\title{
Linear stability of ice growth under a gravity-driven water film
}

\author{
Evgeniy Shapiro, Sergei Timoshin ${ }^{\dagger}$
}

August 17, 2006

\begin{abstract}
In this paper we consider linear stability of ice growth under a gravity driven water film on a sloping wall. First, we derive an analytic solution of the stability problem in the longwave limit which shows that the presence of the ice layer generates an additional wave mode. Further, using a longwave solution as an initial guess, we find the additional wave mode in the numerical solution of the complete Orr-Sommerfeld problem and investigate its behavior numerically for a wide range of problem parameters. We show that the ice mode can become unstable even at moderate Reynolds numbers, and that the ice layer alters the behavior of the mode corresponding to the waves on the liquid film surface. We also demonstrate that the presence of the ice layer stabilizes wave disturbances on the water surface and that, depending on the angle of the incline, the critical Reynolds number of the surface mode can be either increased or decreased.
\end{abstract}

${ }^{*}$ Department of Aerospace Sciences, Cranfield University, UK
${ }^{\dagger}$ Department of Mathematics, University College London, UK 


\section{Introduction}

The study we present in this paper was initially motivated by the experimental results reported by Streitz and Ettema ${ }^{1}$, who performed a series of experiments on the development of an ice layer under a water film flowing down an inclined plane. The dynamics of ice growth suggested that the ice surface might become unstable under certain conditions. The development of the ice surface in this flow bears resemblance to the ice-band structures observed experimentally in the pipe flows by Gilpin ${ }^{2,3}$ and Seki et al. ${ }^{4}$.

Morphological instabilities of the solidification front have been studied extensively in relation to concentration field driven casting problems ${ }^{5,6,7}$. In these problems, the interaction between the flow field and the morphological instability of the solidification front can cause growth and upstream propagation of the solidification front disturbances. For water film flows an asymptotic triple-deck theory was developed by Rothmayer and Tsao ${ }^{8,9,10}$ in order to analyze ice formation on an airfoil at large Reynolds numbers. They found that the ice front can become unstable under the conditions of simultaneous cooling of both air and airfoil surface. In another work ${ }^{11}$, the same authors have demonstrated successful comparisons betwen the triple-deck theory and experimental observations.

A number of researchers studied the stability of gravity-driven water film flow on a sloping wall, starting with a pioneering work by $\mathrm{Yih}^{12}$. In the longwave limit, the linear stability problem for this flow can be solved analytically ${ }^{12}$. This provides, in conjunction with several numerical studies ${ }^{13,14,15}$, an excellent starting point for the analysis of the icing instability and the ice layer influence on the other modes present in the flow, namely the Tollmien-Schlichting and surface waves.

Furthermore, in the triple-deck of Rothmayer and Tsao ${ }^{9}$ icing instability is observed for supercooled water, whereas for water film flow on a sloping wall with air temperature above freezing there exists a steady state solution of the Navier-Stokes and energy equations, which makes it possible to perform a linear stability analysis in a consistent manner.

In this paper we analyze the flow of water film down an inclined plane where the temperature of the plane is below freezing and the temperature of the water surface is above freezing. We formulate a linear stability problem for the flow and derive an analytic solution in the longwave limit. Using this solution, we show that an additional mode appears in the problem and demonstrate that this mode is associated with the presence of the ice layer. Then we proceed to analyze the behavior of this mode using numerical solution of the linear stability problem for order-one wave numbers and show that this mode becomes unstable under certain conditions. It is worth noting, that phase speed of the icing instability mode described in this paper is always negative when this mode is unstable.

Forth and Wheeler ${ }^{6}$ demonstrated that for the casting problems in the boundary layer flow, the effect of 
the solidification front on the hydrodynamic stability (shear instability in their case) is very weak because of the mismatch in the characteristic length scales involved. However for the flow down an inclined plane, the neutral curve of the surface mode includes the Reynolds number axis ${ }^{12}$. Therefore the flow is unstable to arbitrarily long wave disturbances for any Reynolds number exceeding some critical value. In the long wave limit there is no mismatch between the solidification front length scale and surface waves length scale and the ice layer has a potential to influence the other wave modes present in the flow. We investigate this effect and demonstrate using first our longwave analytic solution and then numerical solution of the complete linear stability problem that the ice layer stabilizes water surface mode in the longwave limit and affects its critical Reynolds number.

\section{Nonlinear problem}

The flow of water down an inclined plane maintained at a temperature below freezing is governed by NavierStokes system of equations coupled with heat transfer equations in water and ice layers.

Let us use superscript "*" to denote values with dimensions, and subscripts $L$ and $I$ to refer to the properties of liquid layer and ice sheet respectively.

In order to simplify comparison with the flow without ice formation, we use nondimensionalization adopted in Floryan et al. ${ }^{15}$. We take the thickness of the unperturbed film $h^{*}$ as the characteristic length and write the characteristic velocity in terms of the acceleration due to gravity $g^{*}$ and the angle of the plane with the horizontal $\phi$, as $U^{*}=g^{*} h^{* 2} \sin \phi /\left(2 \nu_{L}^{*}\right)$. Here $\nu_{L}^{*}$ denotes kinematic viscosity of liquid. The characteristic time is then given by $h^{*} / U^{*}$. The characteristic pressure is $P^{*}=\mu_{L}^{*} U^{*} / h^{*}$, where $\mu_{L}^{*}$ denotes dynamic viscosity of liquid. We define dimensionless temperature as $T=\left(T^{*}-T_{F}^{*}\right) / D T$ where $D T=T_{A}^{*}-T_{F}^{*}$ is the characteristic temperature scale, $T_{F}^{*}$ denotes the freezing temperature and $T_{A}^{*}$ denotes the temperature of the surrounding air, which is assumed to be constant. The origin of the coordinate system is placed at the unperturbed free surface, with $y$-axis pointing downward into the liquid layer and the $x$-axis pointing in the direction of the base flow (see Figure 1). In the scope of this paper we consider consider ice surface development at water time-scale, and characteristic time is given by $t=\frac{h^{*}}{U^{*}}$. Note that another approach can be constructed by considering problem at ice time-scale ${ }^{16}$.

Using these characteristic values and usual notation for velocity and pressure, the governing system of equations can be written in nondimensional form as 


$$
\left\{\begin{array}{c}
\frac{D \vec{V}}{D t}=-\nabla p+\frac{1}{R e} \Delta \vec{V}+\frac{1}{F r} \vec{g} \\
\frac{D \overrightarrow{T_{L}}}{D t}=\frac{1}{R e P r} \Delta T_{L} \\
\frac{\partial T_{I}}{\partial t}=\frac{\chi_{I L}}{R e P r} \Delta T_{I}
\end{array}\right.
$$

where $\chi_{I L}=\chi_{I} / \chi_{L}$ is the ratio of thermal diffusivities and $\overrightarrow{n_{g}}$ indicates the direction of acceleration due to gravity $g$. The Reynolds number is given by $R e=h^{* 3} g^{*} \sin \phi /\left(2 \nu_{L}^{* 2}\right)$, the Prandtl number by $\operatorname{Pr}=\nu_{L}^{*} / \chi_{L}^{*}$ and the Froude number by $\mathrm{Fr}=4 \nu_{L}^{* 2} /\left(g^{*} h^{* 3} \sin ^{2} \phi\right)$.

Let the position of the free surface be given by equation $F(x, y, t)=0$ and the position of the ice surface by $H(x, y, t)=0$. Then boundary conditions at the free surface are given by kinematic condition, stress balance condition and constant temperature condition

$$
\left\{\begin{array}{c}
\vec{V} \cdot \nabla F=-\frac{\partial F}{\partial t} \\
\left(-p \cdot \delta_{i j}+\left(\frac{\partial V_{i}}{\partial x_{j}}+\frac{\partial V_{j}}{\partial x_{i}}\right)\right) \cdot n_{j}^{F}+\frac{R e}{W e} \kappa n_{i}^{F}=0 \\
T_{L}=1
\end{array}\right.
$$

where $n_{i}^{F}$ denotes components of the unit normal vector to the free surface, $W e=\rho_{L}^{*} g^{* 2} h^{* 5} \sin ^{2} \phi /\left(4 S^{*} \nu_{l}^{* 2}\right)$ is the Weber number, $S^{*}$ is the surface tension coefficient and $\kappa$ is the curvature of the free surface.

Boundary conditions at the ice surface are given by the kinematic condition adjusted to account for the volume change accompanying phase change, Stefan condition ${ }^{17}$ representing energy conservation and constant freezing temperature condition

$$
\left\{\begin{array}{c}
\vec{V} \cdot \nabla H=-\Theta_{I L} \cdot \frac{\partial H}{\partial t} \\
\lambda_{I L} \frac{\partial T_{I}}{\partial n^{\vec{H}}}-\frac{\partial T_{L}}{\partial n^{\vec{H}}}=\frac{R e P r}{S t} \vec{V} \cdot \overrightarrow{n^{F}} \\
T_{L}=T_{I}=0
\end{array}\right.
$$

where $\overrightarrow{n^{H}}$ denotes the unit normal vector to the ice surface, $\Theta_{I L}=1-\rho_{I}^{*} / \rho_{L}^{*}$ is a positive constant involving only the densities of the ice $\left(\rho_{I}^{*}\right)$ and water $\left(\rho_{L}^{*}\right)$ and $\lambda_{I L}=\lambda_{I}^{*} / \lambda_{L}^{*}$ is the ratio of ice thermal conductivity to water thermal conductivity. Stefan number is defined as $S t=C p_{L}^{*} D T / H_{L I}^{*}$ using water specific heat $C p_{L}^{*}$ and latent heat of the phase change $H_{L I}^{*}$. Quantitative estimates performed in this paper are based on $C p_{L}^{*}=4218 \mathrm{~J} / \mathrm{kg} \cdot \mathrm{K}$ and $H_{L I}^{*}=333.4 \mathrm{~kJ} / \mathrm{kg}$ (see, for example ${ }^{17,18}$ ).

At solid wall we assume constant temperature $T_{W}$

$$
T_{I}=T_{W}=\text { const }
$$

Note that Eqs. (1) do not include the effect of buoyancy. In the Boussinesque approximation, the relative 
magnitude of buoyancy term to viscous term is given by

$$
R(D T, \phi)=\frac{2 \beta D T}{\sin (\phi)}
$$

where $\beta$ is the volumetric expansion coefficient of water. In our case we consider small variations of temperature $\left(D T<5^{\circ}\right)$ and the minimum angle of incline considered in this paper is equal 0.002 . Using fourth order approximation for water density as a function of temperature ${ }^{19}$, we can estimate the maximum of $R$ to be $R(5,0.002)=-0.079$. Based on this estimate we have chosen to neglect buoyancy effects in all cases presented in this paper. However it is worth noting that for smaller angles of incline, bouyancy should be included into the problem formulation.

\section{Linear stability problem}

Consider linear stability problem for the system of Eqs. (1) -(4). The base steady-state solution of Eqs. (1) -(4) independent of the $x$-coordinate is given by a half-parabolic velocity profile and linear temperature distributions in both liquid and solid phases, which can be written as

$$
\left\{\begin{array}{c}
u_{0}(y)=1-y^{2} \\
v_{0}(y)=0 \\
p_{0}(y)=2 y \cot \phi \\
T_{0 L}(y)=1-y \\
T_{0 I}(y)=T_{W} \frac{y-1}{H_{0}-1}
\end{array}\right.
$$

where $y=H_{0}$ gives the coordinate of the cold wall.

Using the normal modes method, we consider evolution of small traveling wave disturbances to the base flow (Eq. (6)) which are defined for each flow variable $F$ as $F(x, y, t)=\widehat{F}(y) \exp (i \alpha(x-c t))$, where $\alpha$ is the real wave number and $c$ is the complex phase speed. The resulting Orr-Sommerfeld equations can be formulated in terms of the perturbations of vertical velocity $\widehat{v}$ and temperature in the water layer $\widehat{T_{L}}$

$$
\left\{\begin{array}{c}
\frac{d^{4} \widehat{v}}{d y^{4}}+\left(-2 \alpha^{2}+i \alpha \operatorname{Re}\left(c-u_{0}\right)\right) \frac{d^{2} \widehat{v}}{d y^{2}}+ \\
\left(\alpha^{4}+i \alpha^{3} \operatorname{Re}\left(u_{0}-c\right)+i \alpha \operatorname{Re} \frac{d^{2} u_{0}}{d y^{2}}\right) \widehat{v}=0 \\
\frac{d^{2} \widehat{T_{L}}}{d y^{2}}+\left(-\alpha^{2}+i \alpha \operatorname{Re} \operatorname{Pr}\left(c-u_{0}\right)\right) \widehat{T_{L}}-\operatorname{Re} \operatorname{Pr} \frac{d T_{0 L}}{d y} \widehat{v}=0
\end{array}\right.
$$

The kinematic condition at the free surface leads to the following expression for the perturbation of the free surface $\widehat{f}$, 


$$
\widehat{f}=-\frac{i \widehat{v}}{\alpha\left(u_{0}-c\right)}
$$

We write boundary conditions at the unperturbed free surface $y=0$ and neglect the influence of the air flow at the air/water interface. Then using Eq. (8) to eliminate the perturbation of the free surface, we can express continuity of tangential and normal stresses (written at $y=0$ ) as

$$
\left\{\begin{array}{c}
\frac{d^{2} \widehat{v}}{d y^{2}}+\left(\alpha^{2}-\frac{d^{2} u_{0 l}}{d y^{2}} \frac{1}{\left(u_{0 l}-c\right)}\right) \widehat{v}=0 \\
\frac{d^{3} \widehat{v}}{d y^{3}}+\left(-3 \alpha^{2}+i \alpha \operatorname{Re}\left(c-u_{0 l}\right)\right) \frac{d \widehat{v}}{d y}+ \\
i \alpha \frac{\widehat{v}}{\left(u_{0 l}-c\right)}\left(\alpha^{2} \operatorname{Re}^{-\frac{2}{3}}\left(\frac{3}{2} \sin \phi\right)^{-\frac{1}{3}} \zeta+2 \cot \phi\right)=0
\end{array}\right.
$$

where $\zeta=\left(3 \rho_{L}^{*} S^{* 3} /\left(g^{*} \mu_{L}^{* 4}\right)\right)^{\frac{1}{3}}$ is the surface tension parameter which depends only on media properties $^{15}$. In addition, we require the temperature to be constant on the free surface which leads to the following equation

$$
\widehat{T_{L}}-\frac{i \widehat{v}}{\alpha\left(u_{0 l}-c\right)} \frac{d T_{0 L}}{d y}=0
$$

At the ice surface, we write the boundary conditions using expansion at the unperturbed position $y=1$. Then the kinematic condition at the ice surface yields the following expression for the perturbation of the ice surface, $\widehat{h}$,

$$
\widehat{h}=\frac{i \widehat{v}}{\Theta_{I L} \alpha c}
$$

Using Eq. (11) in order to eliminate the perturbation of the ice surface, we can write velocity and temperature conditions on the ice surface as

$$
\frac{d \widehat{v}}{d y}+\frac{\widehat{v}}{\Theta_{I L} c} \frac{d u_{0}}{d y}=0
$$

and

$$
\widehat{T_{L}}+\frac{i \widehat{v}}{\Theta_{I L} \alpha c} \frac{d T_{0 L}}{d y}=0
$$

respectively.

Taking into account linearity of the initial temperature profile, we can write Stefan condition in simplified form as 


$$
\frac{d \widehat{T_{L}}}{d y}-\lambda_{I L} \frac{d \widehat{T_{I}}}{d y}=-\frac{P r \cdot R e}{S t} \widehat{v}
$$

For the ice layer, the normal modes method yields the following boundary-value problem for the disturbance of ice temperature $\widehat{T_{I}}$,

$$
\left\{\begin{array}{c}
\frac{d^{2} \widehat{T_{I}}}{d y^{2}}+\omega_{I}^{2} \widehat{T_{I}}=0 \\
\widehat{T_{I}}\left(H_{0}\right)=0 \\
\widehat{T_{I}}(1)=-\frac{i \widehat{v}(1)}{\Theta_{I L} \alpha c} \frac{d T_{0 I}}{d y}
\end{array}\right.
$$

where $\omega_{I}^{2}=-\alpha^{2}+i \alpha \chi_{L I} c R e P r$. The solution of Eq. (15) is given by

$$
\widehat{T_{I}}(y)=-\frac{i \widehat{v}(1)}{\Theta_{I L} \alpha c} \frac{d T_{0 I}}{d y} \frac{\sinh \left(i \omega_{I}\left(H_{0}-y\right)\right)}{\sinh \left(i \omega_{I}\left(H_{0}-1\right)\right)}
$$

From the energy flux balance written at the ice surface for the unperturbed flow it follows that $H_{0}=$ $1-\lambda_{I L} T_{W}$. Combining this with Eq. (16) we can eliminate the ice temperature from the Stefan condition (Eq. (14)) which then becomes

$$
\frac{d \widehat{T_{L}}}{d y}=\left(\frac{\omega_{I}}{\Theta_{I L} k c} \operatorname{coth}\left(i \omega_{I}\left(H_{0}-1\right)\right)-\frac{\operatorname{Pr} R e}{S t}\right) \widehat{v}
$$

Detailed derivations of the boundary conditions can be found in Shapiro ${ }^{16}$.

\section{Numerical approach}

In order to solve Eq. (7) we discretize the equations and boundary conditions using a compact $4^{\text {th }}$ order accurate finite-difference scheme derived in Shapiro and Timoshin ${ }^{20}$ for linear stability analysis of two-fluid channel flows. Then, following ${ }^{21}$ we diagonalize the matrix of the resulting linear algebraic equations and seek the value of the complex phase speed which reduces the rank of this matrix. Once we have found the solution for a mode at some point in the parametric space $P^{0}=\left(\alpha^{0}, R e^{0}, H_{0}^{0}, S t^{0}, \phi^{0}\right)$, the root for this mode at some other point $P^{1}=\left(\alpha^{1}, R e^{1}, H_{0}^{1}, S t^{1}, \phi^{1}\right)$ is obtained by connecting these points with a line segment in the parametric space and marching along this line with a suitably small step. At each step we use results from the previous step as a guess value for the iterative algorithm. In what follows we refer to this process as mode tracing.

In order to obtain the critical point $P^{c r i t}$, in the $(\alpha, R e)$ plane, we look for the roots of $d R e / d \alpha=0$ equation using Newton iterations. The second derivative $d^{2} R e / d \alpha^{2}$ required for this procedure is computed 
numerically.

In order to test the proposed scheme, we note that if the boundary conditions at the ice surface are replaced with the no-slip conditions corresponding to a fixed solid wall the temperature field becomes decoupled from the disturbance velocity and pressure. In this case the stability modes should be identical to those in the flow down an inclined plane without ice. The results of our tests showed excellent agreement with the data published by De Bruin ${ }^{13}$ and Floryan et al. ${ }^{15}$ for the flow down an inclined plane.

For the computations reported in this paper we use grids of 400 points in the $y$-direction for function plots and grids of 800 points in $y$ for the computation of critical parameters.

\section{Longwave asymptotic solution}

For a liquid film flow on an inclined flat plate, $\mathrm{Yih}^{12}$ derived an analytic solution in the long wave limit. In this section we consider a similar problem in the presence of an ice layer. Apart from being of interest in its own right, the long wave asymptotic solution derived here is used as an initial approximation in the subsequent sections where computations for the full stability problem are described. In order to facilitate comparison with $\mathrm{Yih}^{12}$ we first rewrite Eq. (7) in terms of the stream function disturbance, $\widehat{\psi}$. Let $\alpha \rightarrow 0$ and $\widehat{\psi}=O(1)$ in the Orr-Sommerfeld equations, and consider the following expansion $\widehat{f}=\widehat{f^{0}}+\alpha \widehat{f^{1}}+\ldots$ for all flow variables. Then, as $\widehat{\psi}=i \widehat{v} / \alpha$, Eqs. (7) written for the $O(1)$ terms in the disturbance stream function and temperature become (omitting superscript 0 )

$$
\left\{\begin{array}{c}
\frac{d^{4} \widehat{\psi}}{d y^{4}}=0 \\
\frac{d^{2} \widehat{T_{L}}}{d y^{2}}=0
\end{array}\right.
$$

with the boundary conditions given by

$$
\left\{\begin{array}{c}
\frac{d^{3} \widehat{\psi}}{d y^{3}}=0 \\
\widehat{T_{L}}-\frac{d T_{0 L}}{d y} \frac{1}{u_{0}-c} \widehat{\psi}=0 \\
\frac{d^{2} \widehat{\psi}}{d y^{2}}-\frac{d^{2} u_{0}}{d y^{2}} \frac{1}{u_{0}-c} \widehat{\psi}=0
\end{array}\right.
$$

at the free surface, and 


$$
\left\{\begin{array}{c}
\frac{d \widehat{\psi}}{d y}+\frac{d u_{0}}{d y} \frac{1}{\Theta_{I L} c} \widehat{\psi}=0 \\
\widehat{T_{L}}+\frac{d T_{0 L}}{d y} \frac{1}{\Theta_{I L} c} \widehat{\psi}=0 \\
\frac{d \widehat{T_{L}}}{d y}-\lambda_{I L} \frac{d \widehat{T_{I}}}{d y}=i \alpha \frac{\operatorname{PrRe}}{S t} \widehat{\psi}
\end{array}\right.
$$

at the ice surface respectively. The longwave asymptotic solution is valid if $\alpha \ll 1$ and $\alpha R e \ll 1$. However in addition, we require that the coefficient at $\widehat{\psi}$ in the Stefan condition $\alpha \operatorname{Re} \operatorname{Pr} / S t \sim 1$. This condition is necessary in order to retain the influence of the ice surface deformations in the limit formulation. Let us denote that coefficient by $a$ and estimate the validity of these assumptions. For water near freezing temperature $\operatorname{Pr} \sim 10$, also for characteristic temperature differences of order 1 Stefan number $S t \sim 10^{-2}$ so that $a \sim 10^{3} \alpha R e$. Therefore the assumption $a \sim 1$ holds for $\alpha R e \sim 10^{-3}$. Note when we assume $a \sim 1$, for a given Reynolds number this imposes a window in $\alpha$ in which the comparison between longwave asymptotics and full Orr-Sommerfeld solution can be performed.

The general solution of the system of Eq. (18) (taking into account $d^{3} \widehat{\psi} / d y^{3}=0$ boundary condition from Eq.(19)) is given by

$$
\left\{\begin{array}{c}
\widehat{\psi}=C_{2} y^{2}+C_{1} y+C_{0} \\
\widehat{T_{L}}=C_{4} y+C_{3}
\end{array}\right.
$$

Also the solution for the ice temperature is obtained immediately in the form

$$
\widehat{T_{I}}=\widehat{\psi}(1) \frac{H_{0}-y}{H_{0}-1} \frac{\lambda_{L I}}{\Theta_{I L} c}
$$

The boundary conditions (given by Eqs. (19)-(20)) lead to a linear system for the unknown constants $C_{1-4}$ with the system matrix of the form

$$
A=\left(\begin{array}{ccccc}
0 & 1 & 0 & 0 & -\frac{d T_{0 L}}{d y}(0) \frac{1}{u_{0 l}-c} \\
0 & 0 & 2 & 0 & -\frac{d^{2} u_{0 l}}{d y^{2}}(0) \frac{1}{u_{0}(0)-c} \\
0 & 0 & 2+\frac{d u_{0 l}}{d y}(1) \frac{1}{\Theta_{I L} c} & 1+\frac{d u_{0 l}}{d y}(1) \frac{1}{\Theta_{I L} c} & \frac{d u_{0 l}}{d y}(1) \frac{1}{\Theta_{I L} c} \\
1 & 1 & \frac{d T_{0 L}}{d y}(1) \frac{1}{\Theta_{I L} c} & \frac{d T_{0 L}}{d y}(1) \frac{1}{\Theta_{I L} c} & \frac{d T_{0 L}}{d y}(1) \frac{1}{\Theta_{I L} c} \\
1 & 0 & \frac{1}{H_{0}-1} \frac{1}{\Theta_{I L} c}-i a & \frac{1}{H_{0}-1} \frac{1}{\Theta_{I L} c}-i a & \frac{1}{H_{0}-1} \frac{1}{\Theta_{I L} c}-i a
\end{array}\right)
$$

A non-trivial solution exists if the determinant of the matrix $A$ vanishes. This yields the following quadratic equation for the complex phase speed $c$ 


$$
i a c^{2}\left(H_{0}-1\right) \Theta_{I L} c^{2}-c\left(2 i a \Theta_{I L}\left(H_{0}-1\right)+H_{0}-\Theta_{I L}\left(H_{0}-1\right)\right)+2=0
$$

From here, we obtain two distinct wave modes with the phase speeds given by

$$
\begin{gathered}
c_{ \pm}=\frac{\left(2 i a \Theta_{I L}\left(H_{0}-1\right)+H_{0}-\Theta_{I L}\left(H_{0}-1\right)\right) \pm}{4 i a\left(H_{0}-1\right) \Theta_{I L}} \\
\frac{\sqrt{\left(2 i a \Theta_{I L}\left(H_{0}-1\right)+H_{0}-\Theta_{I L}\left(H_{0}-1\right)\right)^{2}-8 i a\left(H_{0}-1\right) \Theta_{I L}}}{4 i a\left(H_{0}-1\right) \Theta_{I L}}
\end{gathered}
$$

For given media properties, the functions $c_{ \pm}(a)$ reflect the dependence of the phase speed on the wave number when the Reynolds number in the flow is kept constant. We can then use plots of this function for varying initial ice layer height $H_{0}$ (Figure 2) to illustrate the behavior of long waves. Both wave modes appear to be stable and when $H_{0} \rightarrow 1$, i.e. when the ice sheet vanishes, we have $c=2$ as in Yih $^{12}$ (with a rescaling applied as a different normalization of the base-flow solution is taken here). Also both roots converge to Yih's root when $a \rightarrow 0$ and $\Theta_{I L} \rightarrow 0$, i.e. when the coupling between the energy and momentum equations vanishes.

Thus instead of one neutrally-stable surface mode as obtained by $\mathrm{Yih}^{12}$, the longwave stability formulation including ice/water interaction provides two decaying modes. When these modes are used to provide an initial guess for the numerical solution of Orr-Sommerfeld formulation, it occurs that the longwave root $c_{+}$forms a neutral curve for the surface waves whereas the root $c_{-}$continues parametrically into a new mode of instability which we will refer to as the ice mode.

To illustrate this correspondence we compare the behavior of the complex phase speed as a function of wave number for longwave roots with computed eigenvalues of the full Orr-Sommerfeld problem. The comparisons are performed at a fixed angle of incline $(\phi=0.002)$, Stefan number $(S t=0.0625)$ and Prandtl number $\operatorname{Pr}=13.47$. The Orr-Sommerfeld solutions were computed at $R e=10000$ for the undisturbed ice layer height $H_{0}=10$ with zero surface tension. Figure 3 shows imaginary and real parts of $c_{ \pm}$and the corresponding modes from the full Orr-Sommerfeld formulation. In Figures $3 \mathrm{a}, \mathrm{b}$ the longwave mode $c_{-}$is shown together with the ice mode obtained from the solution of the Orr-Sommerfeld problem. Figures 3c,d present a comparison between the longwave mode $c_{+}$and the surface mode in the full formulation. The surface mode was identified by tracing it from the longwave limit to $\alpha=1 \mathrm{using}$ also the data presented in Floryan et al. ${ }^{15}$ for additional verification. Note that the comparison between the real part $c_{+}$mode and surface mode is not as favourable as other comparisons presented on Figure 3. A possible reason for this discrepancy is that ideally, in order to keep the coefficient in the Stefan condition, it is necessary to consider the complete $O(\alpha R e)$ decomposition. However these results allow us to identify the longwave asymptotic modes $c_{-}$and $c_{+}$as the ice mode and the surface mode in the numerical solution of Orr-Sommerfeld problem 
respectively.

From the long wave asymptotic solution it is also clear that the ice layer affects the behavior of waves on the water surface. Indeed, without the ice layer the surface mode is unstable in the longwave limit at such Reynolds numbers (the critical Reynolds number for $\phi=0.01$ being 125$)^{12}$. While with the ice layer present, the surface mode is stable in both long wave solution and numerical solution of Orr-Sommerfeld formulation. This effect will be considered in more detail in the next section.

\section{$6 \quad$ Linear stability results}

\subsection{Ice mode instability}

The results obtained from the longwave asymptotic solution provide us with the initial guess values required for the ice and surface mode computations in the full Orr-Sommerfeld formulation. The starting values for one more wave mode present in the flow, due to a Tollmien-Schlichting instability mechanism, were derived from De Bruin's data ${ }^{13}$.

Figure 4 illustrates variations with the wave number of the imaginary and real parts of the complex phase speed, $c_{I}(\alpha)$ and $c_{r}(\alpha)$ respectively, for all three modes at a fairly high but fixed Reynolds number and with the other parameters chosen such that all three modes exhibit instability. One can see that when the ice mode is unstable the real part of the phase speed for this mode is negative. The absolute values of $c_{i, r}$ for the ice mode are much smaller than those of shear and surface modes. This is consistent with the timescale analysis and experimental results as characteristic time of the ice layer growth observed in experiments is much larger than the characteristic time of the flow development.

In order to verify further the physical identity of the third (ice) mode introduced by the ice layer, we performed an additional test computation for the same control parameters but with the wall conditions on the ice surface replaced with the fixed solid wall no-slip requirement. This leads to the interaction between the temperature field and flow field (governed by the Stefan and kinematic conditions at the ice surface) being "turned off" in effect. We found that at least graphically the plots for shear and surface modes in Figure 4 remained unaltered whereas the eigenvalues for the ice mode could not be obtained numerically.

Figure 5a shows typical neutral curves for all three modes in the flow computed for $H_{0}=10, S t=0.0625$ and $\phi=0.002$ in the absence of surface tension. For this set of parameters, as well as for all other cases we have computed the surface mode becomes unstable first. However the ice mode becomes unstable at lower Reynolds numbers than the shear mode. The neutral curve for the ice mode consists of two characteristic branches with the real part of the growth rate always negative inside the ice mode neutral curve. Following 
the results of the longwave asymptotic analysis, we note that in Figure 5a the Reynolds number axis does not belong to the neutral curve of the surface mode so that the surface mode becomes stable in the longwave limit.

Since the media properties are fixed, investigation of the effect of surface tension reduces to two cases only, namely $\zeta=0$ or $\zeta=4899.38$ (see, for example Floryan et al. ${ }^{15}$ ). In the system of governing equations the influence of surface tension is confined to the term $i \widehat{v} \alpha^{3} R e^{-\frac{2}{3}}\left(\frac{3}{2} \sin \phi\right)^{-\frac{1}{3}} \zeta /\left(u_{0 l}-c\right)$ in the normal stress tensor condition (Eq. (9)) on the free surface. The appearance of this term suggests that, for a fixed slope $\phi$, surface tension is only significant at relatively small Reynolds numbers and sufficiently large wave numbers. Accordingly on the computed neutral curves the influence of surface tension is visible on the upper branch of the ice mode neutral curve only (Figure 5b).

Table 1 presents critical parameters for the ice mode with and without surface tension. The critical point for the lower branch of the ice mode neutral curve is found at $R e_{c}=4094.2, \alpha_{c}=0.00696, c_{r c}=-0.001034$ and it is not affected by surface tension within the precision of computations. This result can be expected since the effect of surface tension on waves of that length should be negligible.

Let us consider the effect of the Stefan number on the ice mode for constant water/ice properties and constant values of $\left(H_{0}, \phi, \zeta\right)=(10,0.002,4899.38)$. Since $S t=C p_{L} D T / H_{L I}$, we find that for fixed media properties $S t \simeq 0.01265 D T$ and the Stefan number is determined by the characteristic temperature difference $D T$ only. We will consider the small values of the characteristic temperature difference $D T$ in the range of $[1,5]$, which corresponds to $S t \in[0.01265,0.0625]$. Computations performed for this temperature range indicate that an increase of Stefan number tends to stabilize both branches of the ice mode neutral curve (Figure 6a). The values of critical parameters are summarized in Table 2 for the lower branch and Table 3 for the upper branch respectively.

In order to analyze the influence of the initial ice height $H_{0}$, we need to estimate the range of $H_{0}$. Initial ice height is related to the wall temperature by $H_{0}-1=-\lambda_{I L} T_{W} \simeq 4.0\left(T_{f}^{*}-T_{W}^{*}\right) / D T$. If we fix Stefan number at $S t=0.0625$ (which corresponds to $D T \simeq 5$ ), then $H_{0} \simeq 1+0.8\left(T_{f}^{*}-T_{W}^{*}\right)$ and for reasonable wall temperatures $\left(>-30^{\circ} \mathrm{C}\right)$, the value of $H_{0}$ is in the range $H_{0} \in(1.0,25]$. Figure $6 \mathrm{~b}$ shows critical curves for this range of initial ice height at fixed angle of incline $\phi=0.002$. As the initial ice height decreases, first the lower branch is stabilized followed by stabilization of disturbances near the upper branch. In the opposite limit of increasing ice height the critical parameters on the upper branch tend to an asymptote and critical Reynolds for the lower branch has a minimum (Figure 7). Note that for this angle of incline the critical Reynolds number for the upper branch is always lower then that of the lower branch.

Variation of the angle of incline has a significant influence on the neutral curve of the surface wave mode ${ }^{15}$ and we expect similar behavior for the ice mode. We consider variation of the angle of incline for a fixed 
Stefan number $(S t=0.0625)$, fixed initial ice height $\left(H_{0}=10\right)$ with the surface tension coefficient equal to 4899.38. The choice of the initial ice height is motivated by the results obtained for the varying $H_{0}$ which indicate that the ice mode is more unstable at large values of $H_{0}$ and the critical Reynolds number tends to a limit value as $H_{0}$ increases.

Our computations show that similarly to the behavior of the surface mode, the decrease of the angle of incline tends to stabilize both branches of the ice mode neutral curve (Figure 8a). Critical Reynolds number decreases rapidly as we increase the angle of incline (Figure 8b), however it always stays higher than that of the surface mode. It is interesting that, at sufficiently small angles, the ice mode becomes unstable to disturbances of $O(1)$ wave length earlier than to longwave disturbances, however as the angle of incline increases this behavior changes and longwave instability becomes dominant. This means that although the critical Reynolds number is a continuous function the angle of incline, both the critical wave number and critical phase speed are discontinuous in the parametric space of the problem (Figures $8 \mathrm{c}$ and $8 \mathrm{~d}$ ).

\subsection{The influence of ice on shear and surface modes}

Our computations indicate that the ice layer has no significant influence on the shear mode. Even in the longwave limit, the effect is limited to slight decrease in the real part of the phase speed (see Figure 9), however this decrease becomes discernible only at very small wave numbers where the shear mode is stable.

Initial observations made in the longwave asymptotic solution suggest that the interaction between the ice layer and the flow stabilizes long surface waves. This feature is observed in numerical solution of the Orr-Sommerfeld problem as well. Figure 10 shows $c_{I}(\alpha)$ for the surface mode computed with and without ice layer with fixed $S t=0.0625, H_{0}=10$, and $\phi=0.002$ and various Reynolds numbers. From these results it can be seen that the stabilizing effect is greater at lower Reynolds numbers.

In the flow down an inclined plane without solidification, the neutral curve for the surface mode intersects with the Reynolds number axis ${ }^{12}$. However when the ice layer is present, the neutral curve of the surface mode has no such intersection and develops a distinct critical point instead. Figure 11 shows neutral curves for the surface mode with and without ice for the same wall slopes as used by Floryan et al. ${ }^{15}$.

The ice layer leads to an increase in the critical Reynolds number of the surface mode for larger angles of incline and a decrease in the critical Reynolds numbers for smaller angles. Figure 12a shows the critical Reynolds number with and without ice layer as a function of the angle of incline in logarithmic scale. Here the angle of incline is given in degrees to simplify comparison with Floryan et al. ${ }^{15}$. Note that while the Reynolds number varies exponentially with the angle of incline without the ice sheet, with the ice sheet present the line deviates from the exponential as we increase the angle. Figure 12b shows critical Reynolds 
number difference defined as

$$
D R e=\frac{R e^{\text {noice }}-R e^{i c e}}{\operatorname{Re}^{\text {noice }}} 100
$$

where $R e^{i c e}$ and $R e^{\text {noice }}$ are computed with and without the ice layer respectively. To quantify the trends indicated above, the borderline between small and large angles corresponds to $\phi=1.44^{\circ}$ (based on linear extrapolation). Figures $12 \mathrm{c}$ and $12 \mathrm{~d}$ show variation of the critical wave number and phase speed respectively with the angle of incline. Note that for the flow without ice, the critical wave number is zero and the critical phase speed is 2.0 .

Tables 4, 5 and 6 show the influence of the Stefan number on the critical Reynolds number, wave number and phase speed respectively. The increase of the Stefan number destabilizes the flow for smaller angles of incline and stabilizes the flow for larger angles. The critical wave number increases steadily as the Stefan number increases. Also there is an increase in the critical wave speed, however the variation is extremely small.

\section{Discussion and conclusions}

In this paper we consider linear stability of a water film flow down an inclined plane covered with a sheet of ice. We present an asymptotic solution of the stability equations in the long wave limit and show that in this limit the presence of an ice layer generates an additional wave mode and modifies the behavior of the surface mode making the latter stable to long wave disturbances. The asymptotic results are then used to identify the ice mode in the numerical solution of the Orr-Sommerfeld problem with the conclusion that this new mode of instability forms a neutral curve consisting of two distinct branches, one corresponding to long waves and another for disturbances with $O(1)$ wavelength. It is interesting to note that the real part of the phase speed for disturbances of the ice mode is negative.

The problem includes a number of free parameters and we present a detailed analysis of the ice mode behavior under variation of these parameters including the influence of the surface tension, Stefan number, initial ice height and angle of incline on the neutral curves and critical parameters of the ice mode. We find that surface tension stabilizes the $O(1)$ branch of the ice mode while having negligible effect on the longwave branch. The increase of the Stefan number tends to stabilize both branches of the ice mode which can be explained by the diminishing effect of the left hand side term in the Stefan condition (Eq. (17)). As the Stefan number tends to infinity the left hand side of Eq. (17 tends to zero, which means that the flow and temperature fields become decoupled and the ice layer no longer affects the flow stability. 
An increase of the unperturbed ice layer height leads to an increase of the critical Reynolds number on both branches of the ice mode neutral curve. The stabilizing effect of increasing ice layer height is more visible on the long wave branch which is in qualitative agreement with the experimental observations made in pipe flows ${ }^{2,3}$ where the wavy ice-band structures are noted only when the ice layer becomes sufficiently thick. The ice mode behavior for varying angle of incline is similar to that of the surface mode with an increase of the angle of incline leading to a decrease of the ice mode critical Reynolds number. The twobranch structure of the ice mode neutral curve leads to an interesting behavior of the ice mode critical wave number and phase speed, which can become discontinuous functions of the angle of incline. For sufficiently large angles, the ice mode can become unstable at moderate Reynolds numbers, however our computations indicate that it becomes unstable at higher Reynolds numbers than the surface mode.

We also examine the effect of ice on the other instabilities present in the flow. Our computations indicate that the effect of icing on the Tollmien-Schlichting mode is negligible, however the presence of an ice layer stabilizes the surface mode in the longwave limit and modifies the topology of the surface mode neutral curve, creating a distinct turning point instead of the bifurcation point observed without an ice layer ${ }^{12}$. Detailed computations indicate that the effect of icing on the surface mode depends strongly on the angle of incline and is destabilizing for sufficiently small $\left(\phi<1.44^{\circ}\right)$ angles of incline and stabilizing for sufficiently large angles of incline $\left(\phi>1.44^{\circ}\right)$.

\section{References}

[1] J.T. Streitz, R. Ettema, "Observations from an aufeis windtunnel", Cold Regions Science and Technology, 34(2):85-96, 2002

[2] R.R. Gilpin. The morphology of ice structure in a pipe at or near transient reynolds numbers. Heat Transfer - San Diego, AIChE Symposium Series 189, 75:89-94, 1979.

[3] R.R. Gilpin. Ice formation in a pipe containing flows in the transition and turbulent regimes. J. Heat Transfer, 103:363-368, 1981.

[4] N. Seki, S. Fukusako, and G.W. Younan. Ice-formation phenomena for water flow between two cooled parallel plates. J. Heat Transfer, 106:498-505, 1984.

[5] M.E. Glicksman, S.R. Coriell, and G.B. McFadden. Interaction of flows with the crystal-melt interface. Ann. Rev. Fluid Mech., 18:307-335, 1986. 
[6] S.A. Forth and A.A. Wheeler. Hydrodynamic and morphological stability of unidirectional solidification of a freezing binary alloy: a simple model. J. Fluid Mech., 202:339-366, 1989.

[7] S.H. Davis. Hydrodynamic interactions in directional solidification. J. Fluid. Mech., 212:241-262, 1990.

[8] A.P. Rothmayer and J.C. Tsao. A mechanism for ice roughness formation on an airfoil leading edge, contributing to glaze ice accretion. Technical Report 98-0485, AIAA, 1998.

[9] A.P. Rothmayer and J.C. Tsao. Triple-deck simulation of surface glaze ice accretion. Technical Report 2000-0234, AIAA, 2000.

[10] A.P. Rothmayer. On the creation of ice surface roughness by interfacial instabilities. Technical Report 2003-972, AIAA, 2003.

[11] A.P. Rothmayer and J.C. Tsao. Transient effects in predicting bulk surface properties for aircraft ice accretion. Technical Report 2001-0677, AIAA, 2001.

[12] C.S. Yih. Stability of flow down an inclined plane. Phys. Fluids, 6(3):321-334, 1963.

[13] G.J. DeBruin. Stability of a layer of liquid flowing down an inclined plane. J. Engng. Math., 8:259-270, 1974.

[14] R.W. Chin, F.H. Abernathy, and J.R. Bertschy. Gravity and shear wave stability of free surface flows. part1: Numerical calculations. J. Fluid Mech., 168:501-513, 1986.

[15] J.M. Floryan, S.H. Davis, and R.E. Kelly. Instabilities of a liquid film flowing down a slightly inclined plane. Phys. Fluids, 30(4):983-989, 1987.

[16] E. Shapiro. Instabilities in multiphase and icing flows. PhD thesis, University of London, 2004.

[17] V. Alexiades and A.D. Solomon. Mathematical modeling of Melting and Freezing Processes. Hemisphere, Washington, DC, 1993.

[18] E.R. Cohen, D.R. Lide, and G.L. Trigg. AIP Physics Desk Reference. Springer Verlag, New York, NY, 2003.

[19] T.A. Kowalewski and M. Rebow. Freezing of water in a differentially heated cubic cavity. Int. J. Comp. Fluid Dyn., 11:193-210, 1999.

[20] E. Shapiro and S. Timoshin. On the patterns of interaction between shear and interfacial modes in plain air-water poiseuille flow. Proc. R. Soc. A-Math. Phys. Eng. Sci., 461:1583-1597, 2005.

[21] L.H. Thomas. The stability of plane poiseuille flow. Phys. Rev., 91(4):780-783, 1953. 


\begin{tabular}{|c|c|c|}
\hline & $\zeta=0$ & $\zeta=4899.38$ \\
\hline \hline$R e_{c}$ & 2884.7 & 3041.4 \\
\hline$\alpha_{c}$ & 0.772 & 0.796 \\
\hline$c_{r c}$ & -0.0000793 & -0.0000753 \\
\hline
\end{tabular}

Table 1: The influence of surface tension on the critical parameters of the ice mode 


\begin{tabular}{|c|c|c|}
\hline Lower branch & $S t=0.01265$ & $S t=0.0625$ \\
\hline \hline$R e_{c}$ & 2925.7 & 3041.4 \\
\hline$\alpha_{c}$ & 0.819 & 0.796 \\
\hline$c_{r c}$ & -0.0000167 & -0.0000753 \\
\hline
\end{tabular}

Table 2: The influence of the Stefan number on the critical parameters of the ice mode lower branch 


\begin{tabular}{|c|c|c|}
\hline Upper branch & $S t=0.01265$ & $S t=0.0625$ \\
\hline \hline$R e_{c}$ & 3196.7 & 4094.2 \\
\hline$\alpha_{c}$ & 0.00619 & 0.00696 \\
\hline$c_{r c}$ & -0.000390 & -0.00103 \\
\hline
\end{tabular}

Table 3: The influence of the Stefan number on the critical parameters of the ice mode upper branch 


\begin{tabular}{|c|c|c|c|c|}
\hline$S t \backslash \phi$ & $1^{o}$ & $2^{o}$ & $3^{o}$ & $5^{\circ}$ \\
\hline \hline 0.01265 & 71.6 & 36.22 & 24.7 & 15.7 \\
\hline 0.0625 & 71.2 & 36.25 & 25.0 & 16.5 \\
\hline
\end{tabular}

Table 4: The influence of the Stefan number on the surface mode critical Reynolds number 


\begin{tabular}{|c|c|c|c|c|}
\hline$S t \backslash \phi$ & $1^{o}$ & $2^{o}$ & $3^{\circ}$ & $5^{\circ}$ \\
\hline \hline 0.01265 & 0.014262 & 0.022308 & 0.027186 & 0.031044 \\
\hline 0.0625 & 0.016952 & 0.028524 & 0.035106 & 0.040934 \\
\hline
\end{tabular}

Table 5: The influence of the Stefan number on the surface mode critical wave number 


\begin{tabular}{|c|c|c|c|c|}
\hline$S t \backslash \phi$ & $1^{\circ}$ & $2^{o}$ & $3^{o}$ & $5^{\circ}$ \\
\hline \hline 0.01265 & 2.00352 & 2.00464 & 2.00571 & 2.00797 \\
\hline 0.0625 & 2.01116 & 2.01408 & 2.01739 & 2.02352 \\
\hline
\end{tabular}

Table 6: The influence of the Stefan number on the surface mode critical wave speed 


\section{Figures}

\section{Captions:}

1. Schematic of the flow

2. The dependence of the longwave modes $c_{ \pm}$on $a$ for various $H_{0}:$ a) $\left.\operatorname{Im}\left(c_{-}\right) ; \mathrm{b}\right) \operatorname{Re}\left(c_{-}\right)$; c) $\operatorname{Im}\left(c_{+}\right)$; d) $\operatorname{Re}\left(c_{+}\right)$.

3. Comparison between longwave asymptotic solution modes and Orr-Sommerfeld modes: a) $\operatorname{Im}\left(c_{-}\right)$ versus ice mode; b) $\operatorname{Re}\left(c_{-}\right)$versus ice mode; c) $\operatorname{Im}\left(c_{+}\right)$versus surface mode; d) $\operatorname{Re}\left(c_{+}\right)$versus surface mode.

4. Comparison between surface, shear and ice modes $\left(R e=10000, \alpha=0.06 H_{0}=10, S t=0.0625\right.$, $\phi=0.002):$ a) $\operatorname{Im}(c) ;$ b) $\operatorname{Re}(c)$.

5. Typical neutral curves for all modes and the influence of surface tension: a) Typical neutral curves without surface tension; b) Influence of surface tension on the ice mode neutral curve.

6. The influence of the Stefan number and initial ice height on the ice mode neutral curve: a) The influence of the Stefan number; b) The influence of the initial ice height.

7. The influence of the initial ice height $H_{0}$ on the ice mode critical parameters: a) Critical Reynolds number; b) Critical wave number.

8. The influence of the angle of incline on the ice mode: a) Neutral curves; b) Critical Reynolds; c) Critical wave number; Critical phase speed.

9. The influence of the ice layer on the shear mode $\left(S t=0.0625, H_{0}=10, R e=10000\right)$ : a) $\left.\operatorname{Im}(c) ; \mathrm{b}\right)$ $\operatorname{Re}(c)$.

10. Longwave stabilization of the surface mode: a) $\operatorname{Im}(c)$ with ice; b) $\operatorname{Im}(c)$ without ice.

11. The influence of the ice layer on the surface mode neutral curves $\left(S t=0.0625, H_{0}=10\right)$ : a) $\phi=4^{o}$; b) $\phi=1^{\circ}$.

12. The influence of the angle of incline on the surface mode critical parameters: a) Critical Reynolds; b) Critical Reynolds difference $D R e ; c)$ Critical wave number; d) Critical phase speed. 


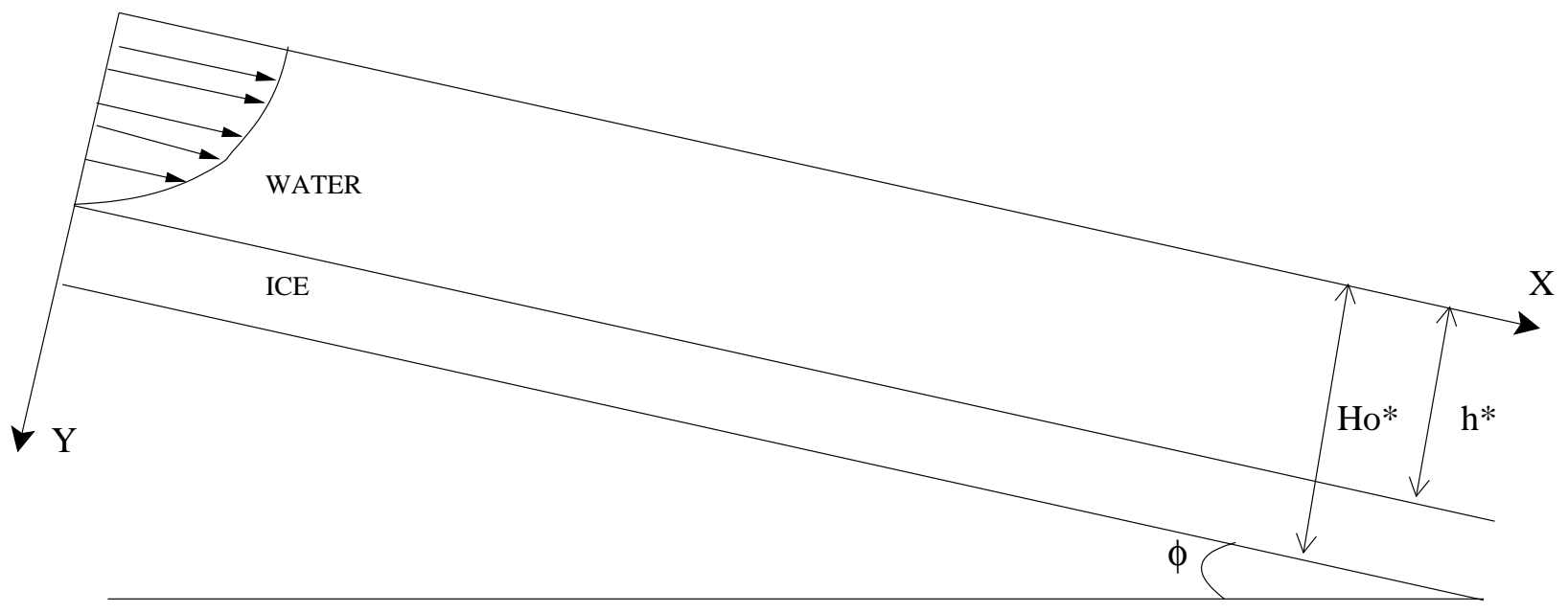

Figure 1: Schematic of the flow 
a)

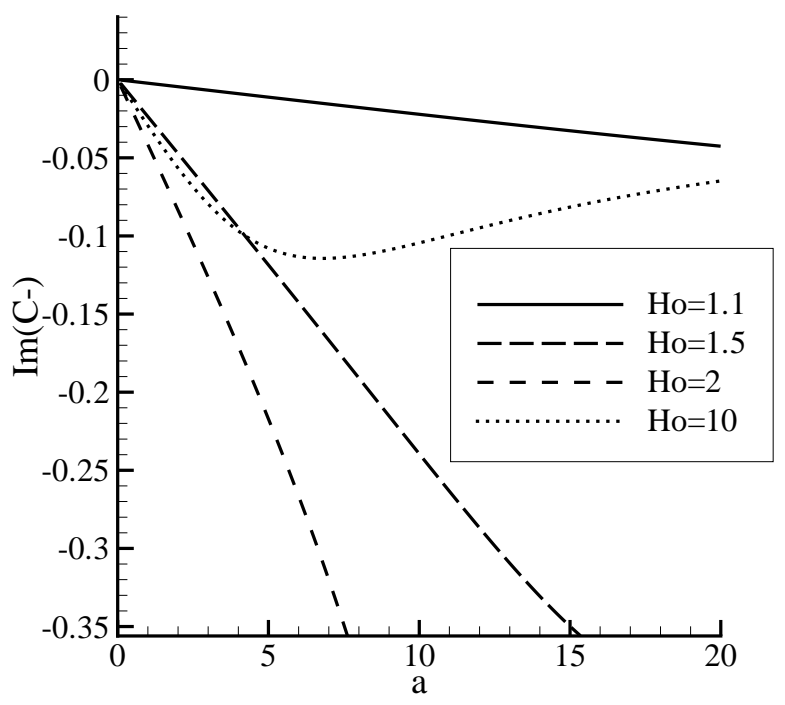

c)

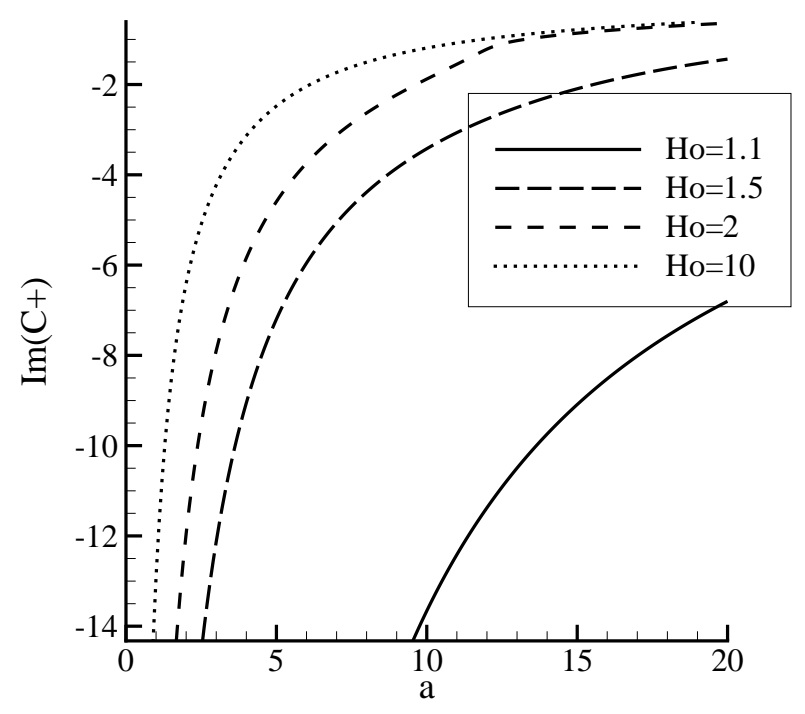

b)

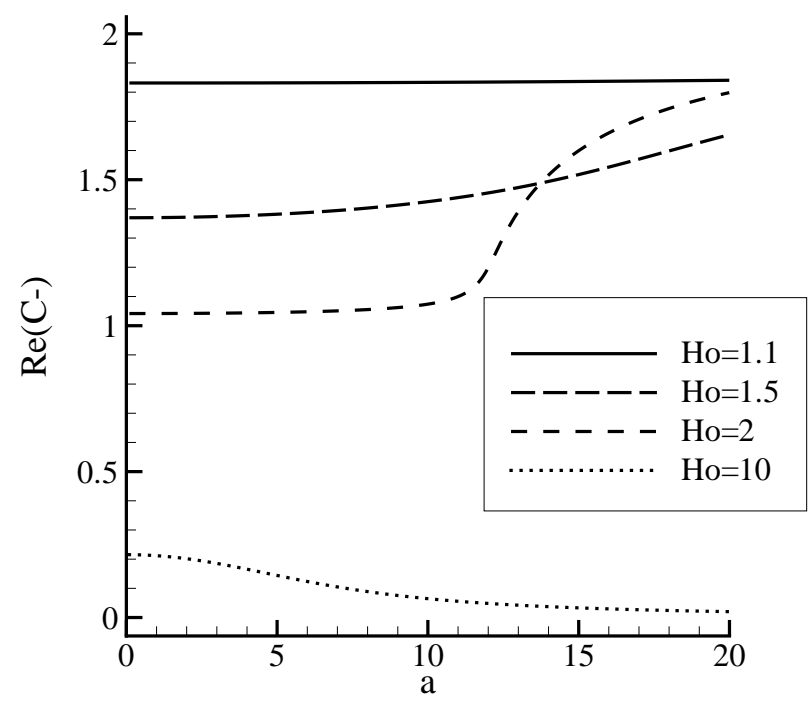

d)

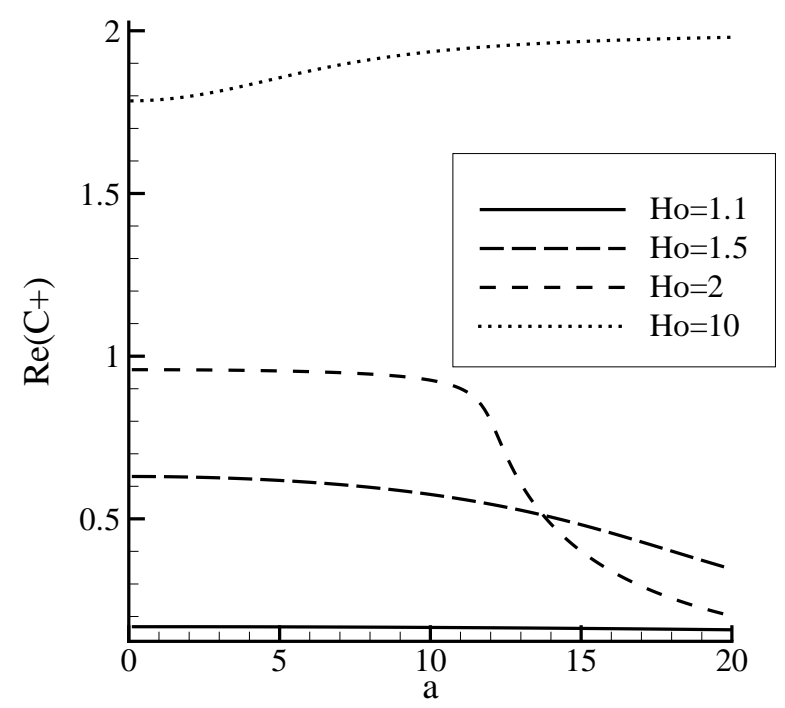

Figure 2: The dependence of the longwave modes $c_{ \pm}$on $a$ for various $H_{0}:$ a) $\left.\left.\operatorname{Im}\left(c_{-}\right) ; \mathrm{b}\right) \operatorname{Re}\left(c_{-}\right) ; \mathrm{c}\right) \operatorname{Im}\left(c_{+}\right)$; d) $\operatorname{Re}\left(c_{+}\right)$. 
a)

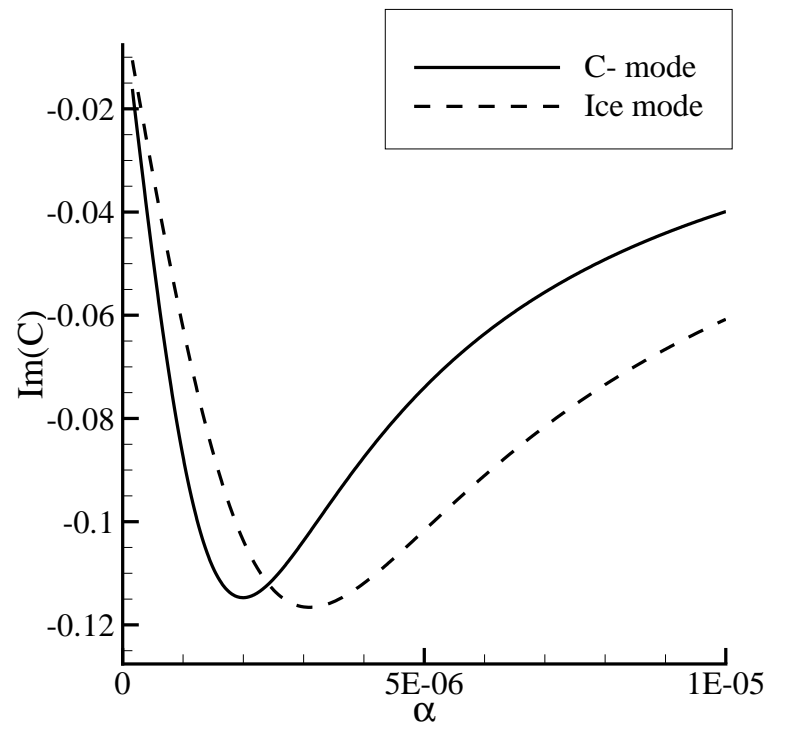

c)

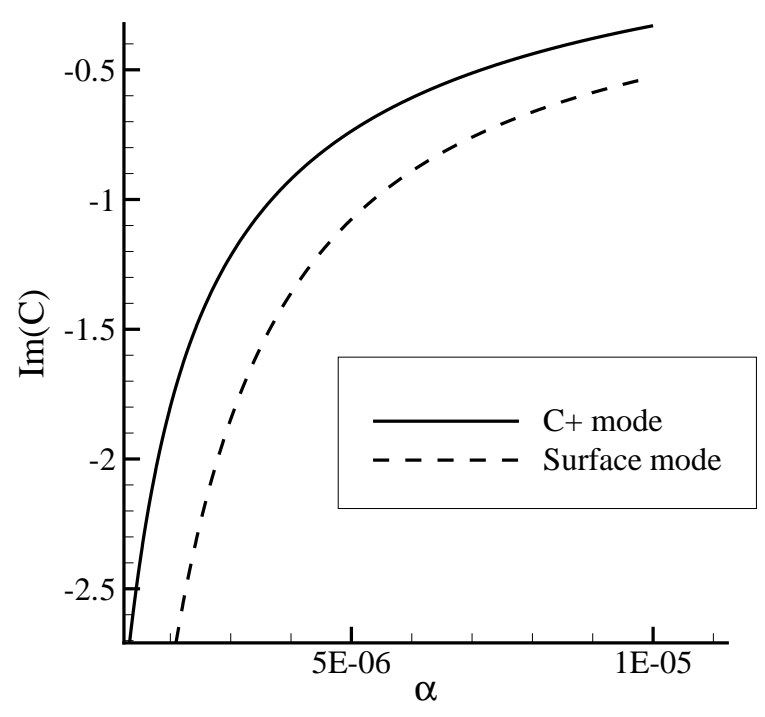

b)

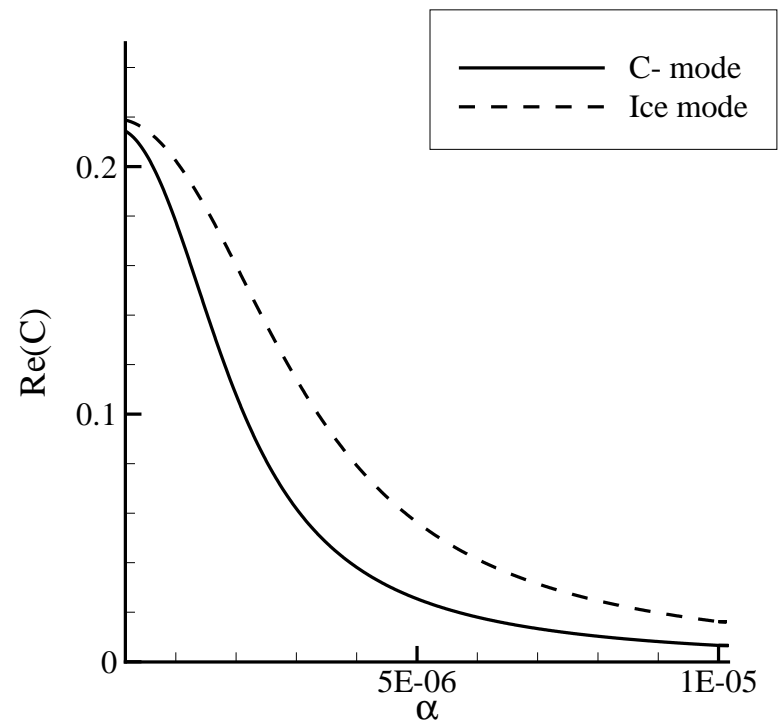

d)

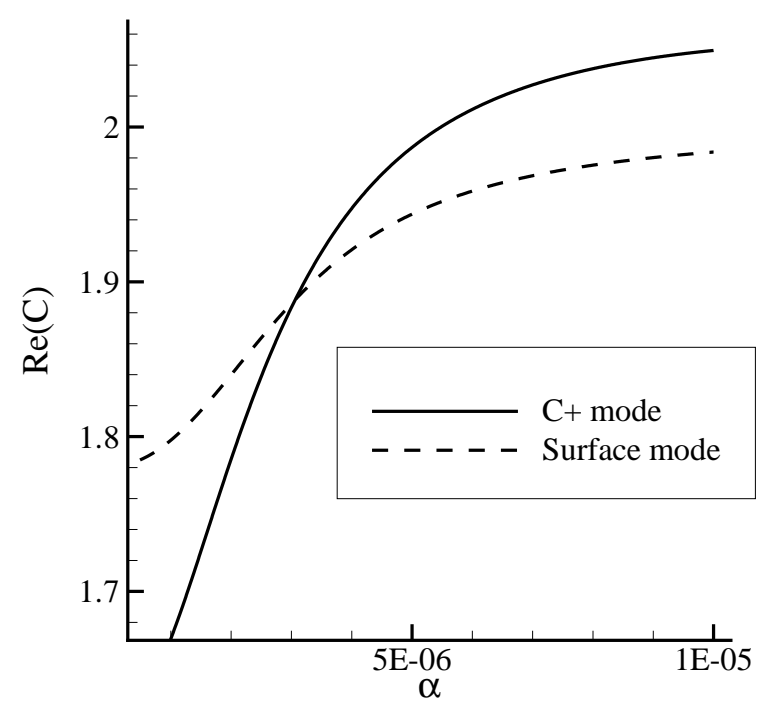

Figure 3: Comparison between longwave asymptotic solution modes and Orr-Sommerfeld modes: a) $\operatorname{Im}\left(c_{-}\right)$ versus ice mode; b) $\operatorname{Re}\left(c_{-}\right)$versus ice mode; c) $\operatorname{Im}\left(c_{+}\right)$versus surface mode; d) $\operatorname{Re}\left(c_{+}\right)$versus surface mode. 

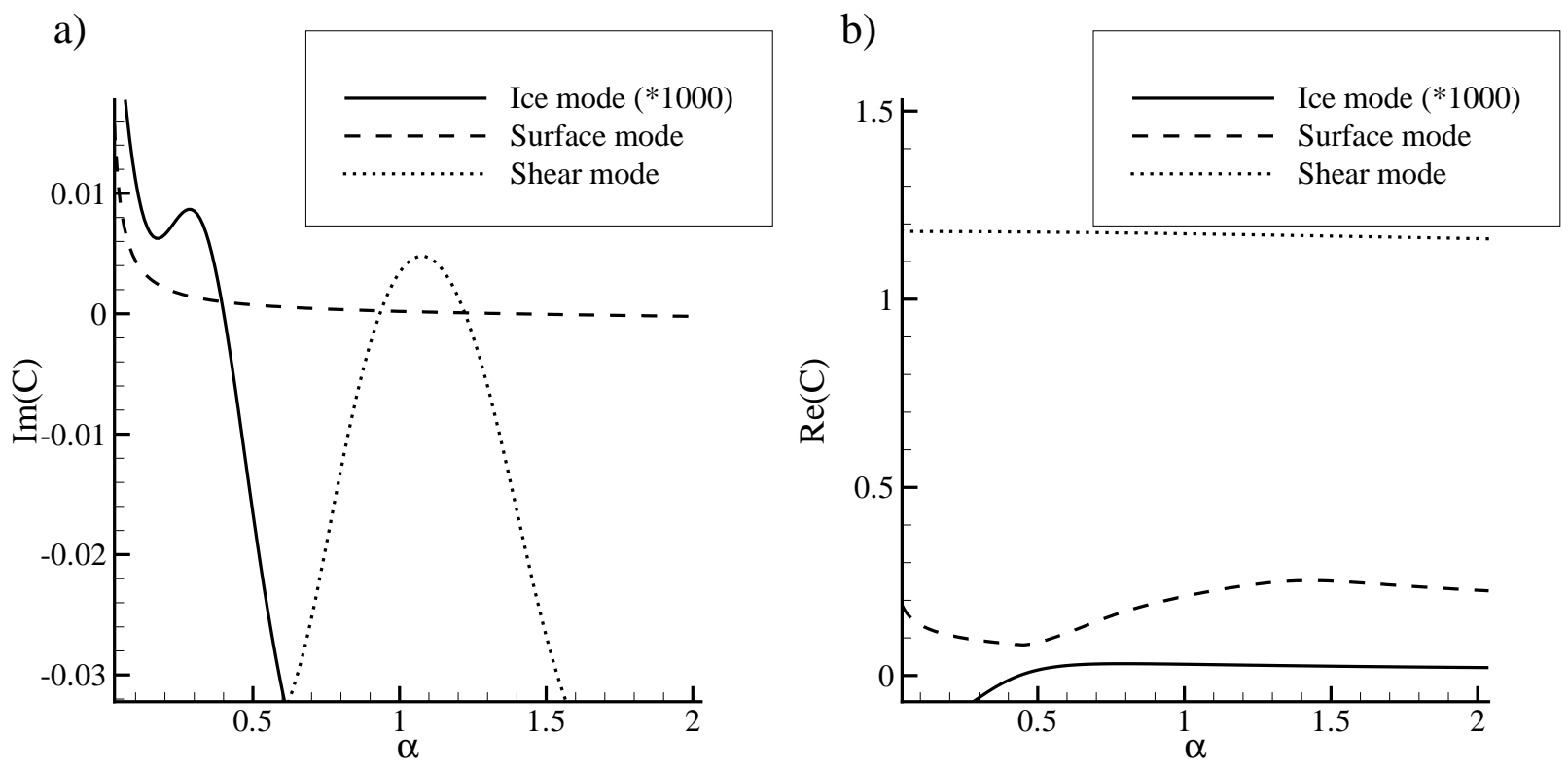

Figure 4: Comparison between surface, shear and ice modes $\left(R e=10000, \alpha=0.06 H_{0}=10, S t=0.0625\right.$, $\phi=0.002)$ : a) $\operatorname{Im}(c) ;$ b) $\operatorname{Re}(c)$.

a)

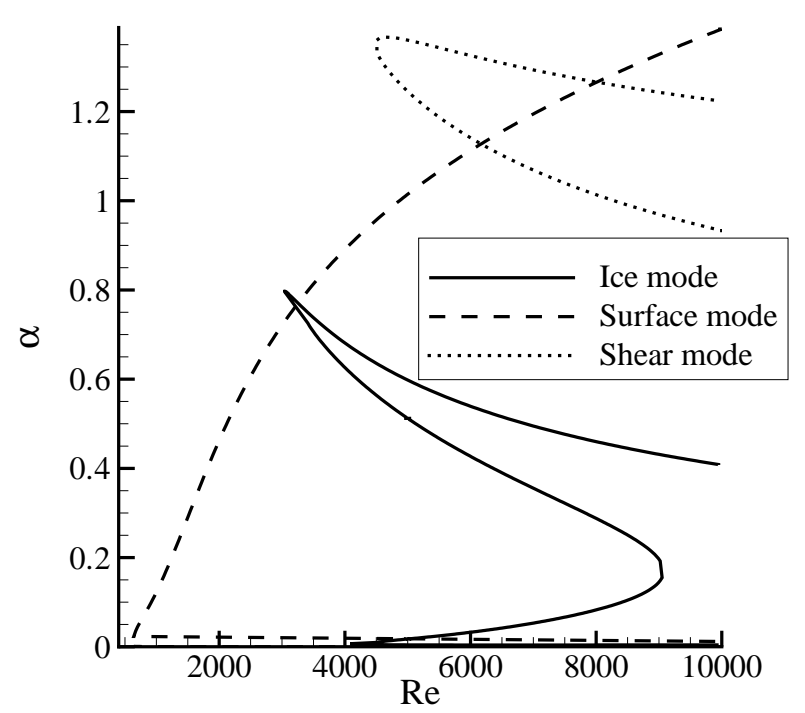

b)

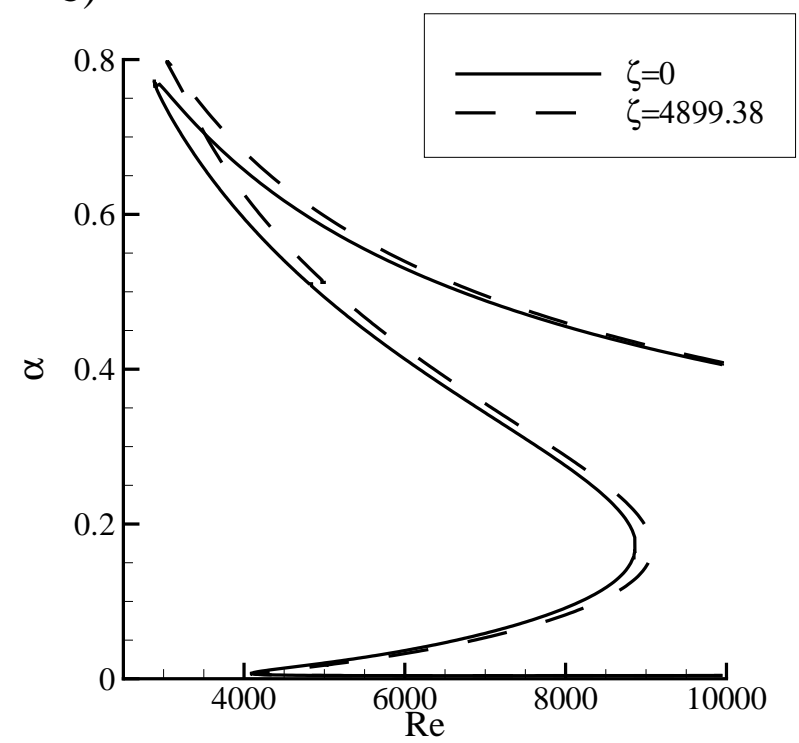

Figure 5: Typical neutral curves for all modes and the influence of surface tension: a) Typical neutral curves without surface tension; b) Influence of surface tension on the ice mode neutral curve. 
a)

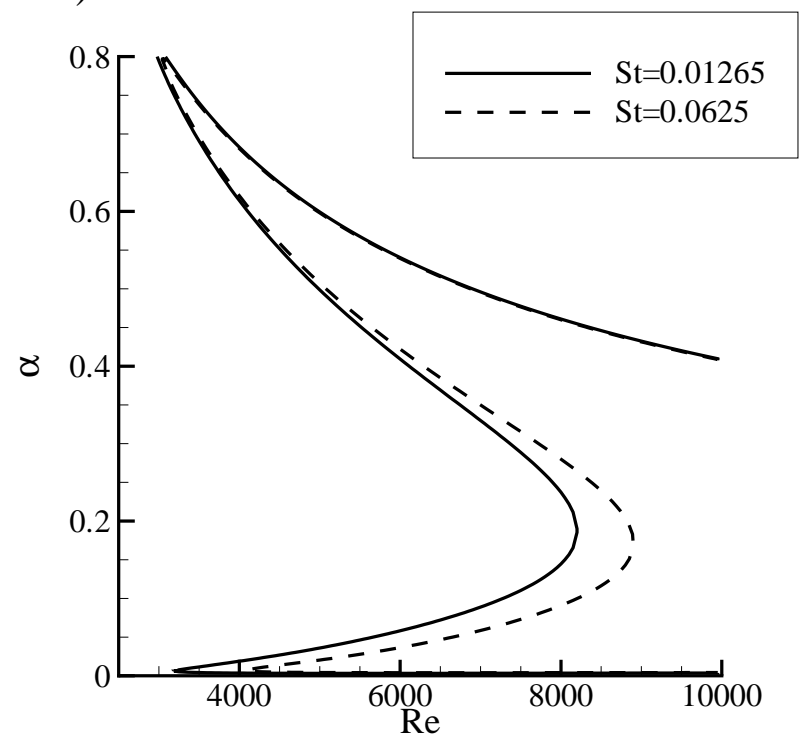

b)

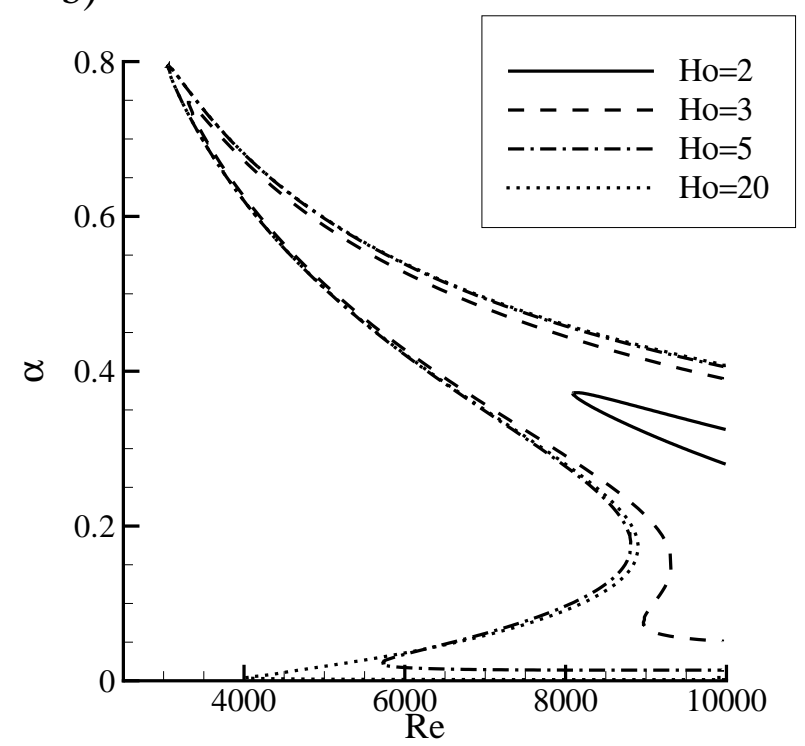

Figure 6: The influence of the Stefan number and initial ice height on the ice mode neutral curve: a) The influence of the Stefan number; b) The influence of the initial ice height.

a)

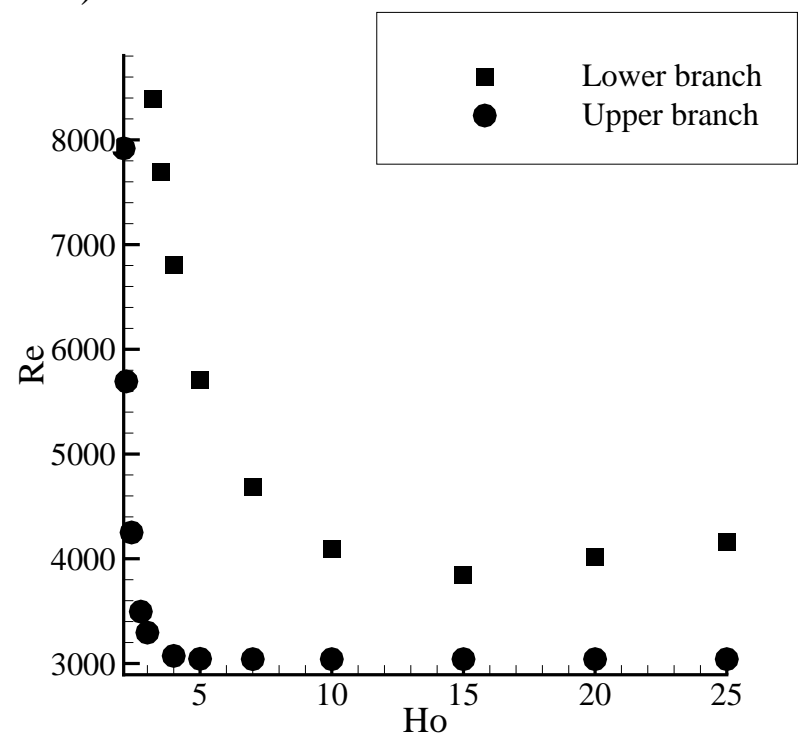

b)

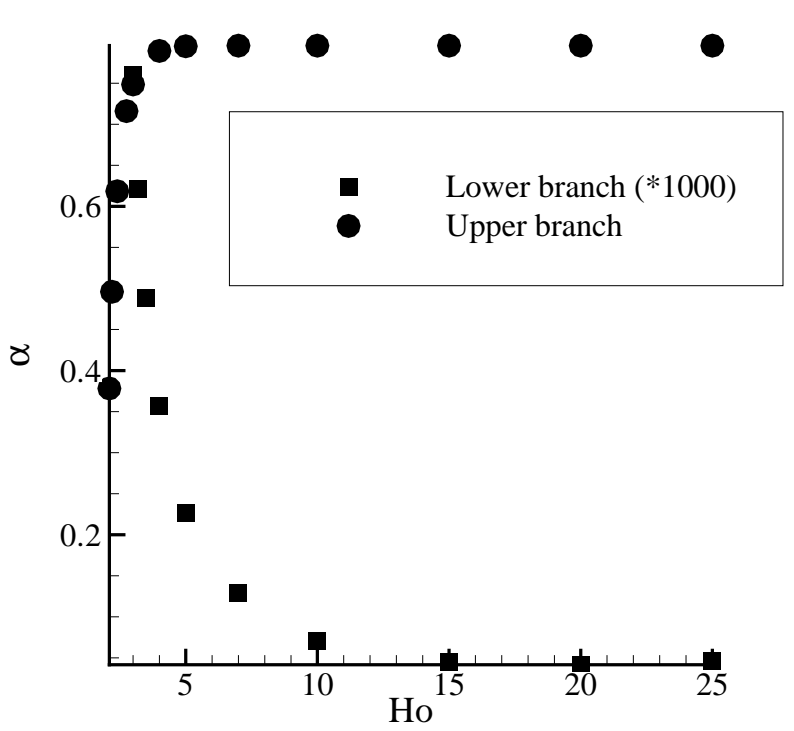

Figure 7: The influence of the initial ice height $H_{0}$ on the ice mode critical parameters: a) Critical Reynolds number; b) Critical wave number. 
a)

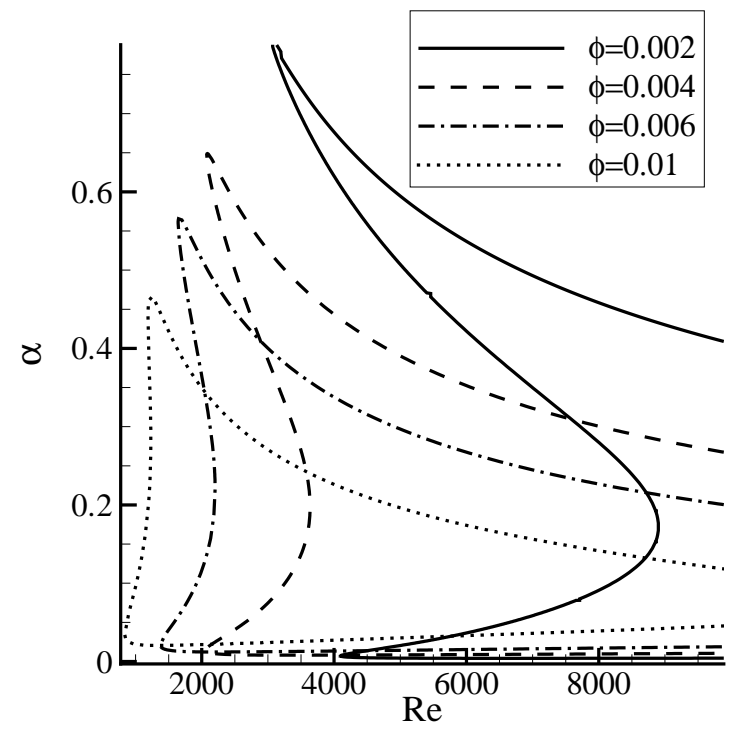

c)

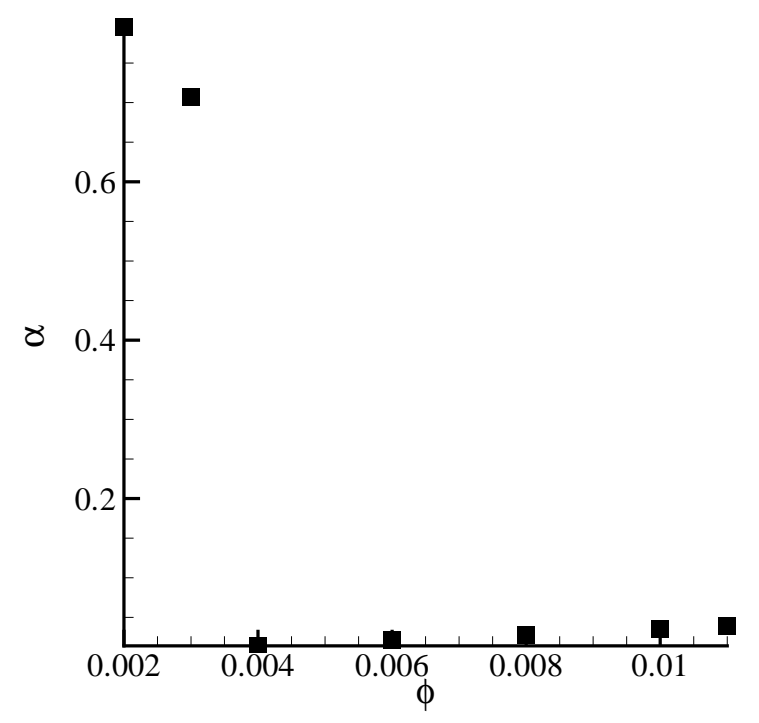

b)

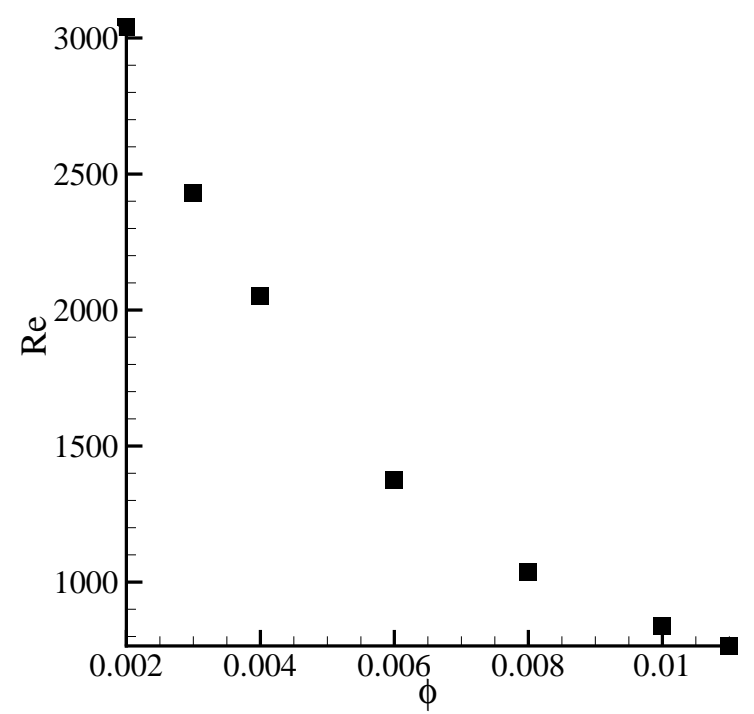

d)

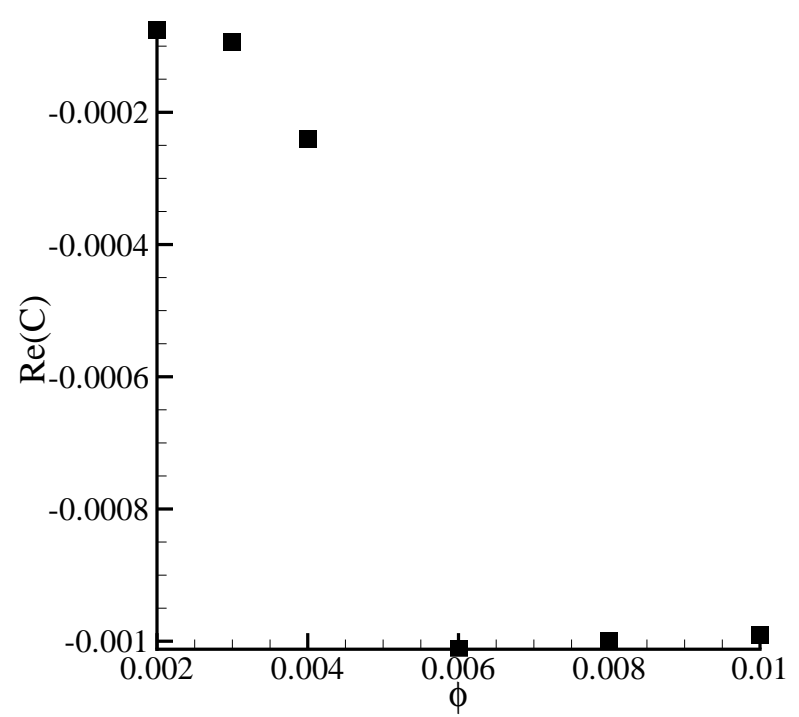

Figure 8: The influence of the angle of incline on the ice mode: a) Neutral curves; b) Critical Reynolds; c) Critical wave number; Critical phase speed. 
a)

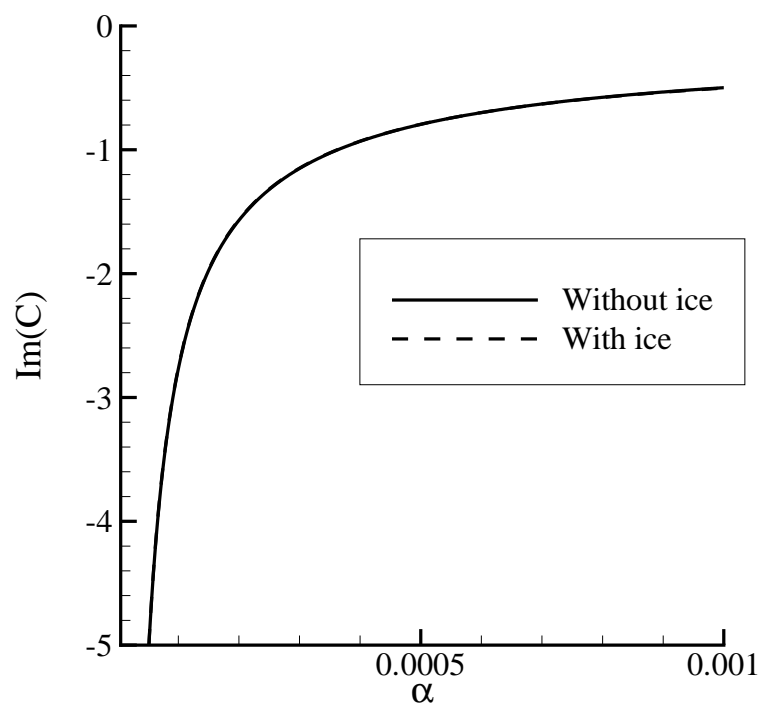

b)

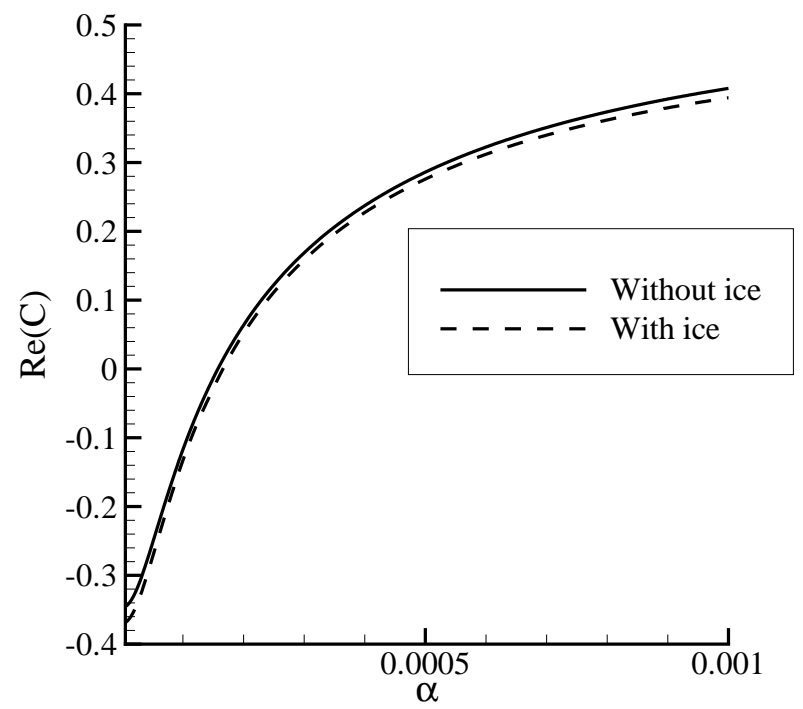

Figure 9: The influence of the ice layer on the shear mode $\left(S t=0.0625, H_{0}=10, R e=10000\right)$ : a) $\operatorname{Im}(c)$; b) $\operatorname{Re}(c)$.

a)

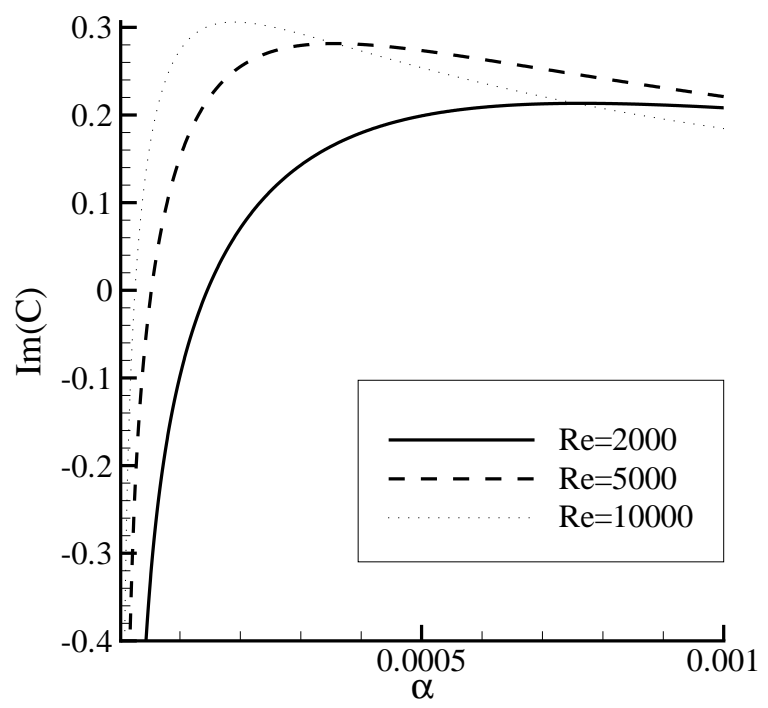

b)

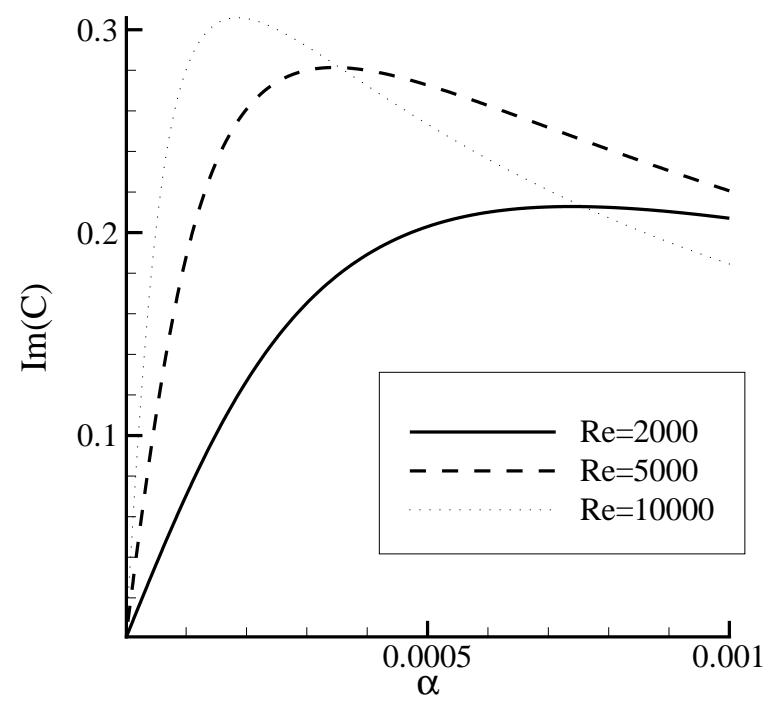

Figure 10: Longwave stabilization of the surface mode: a) $\operatorname{Im}(c)$ with ice; b) $\operatorname{Im}(c)$ without ice. 

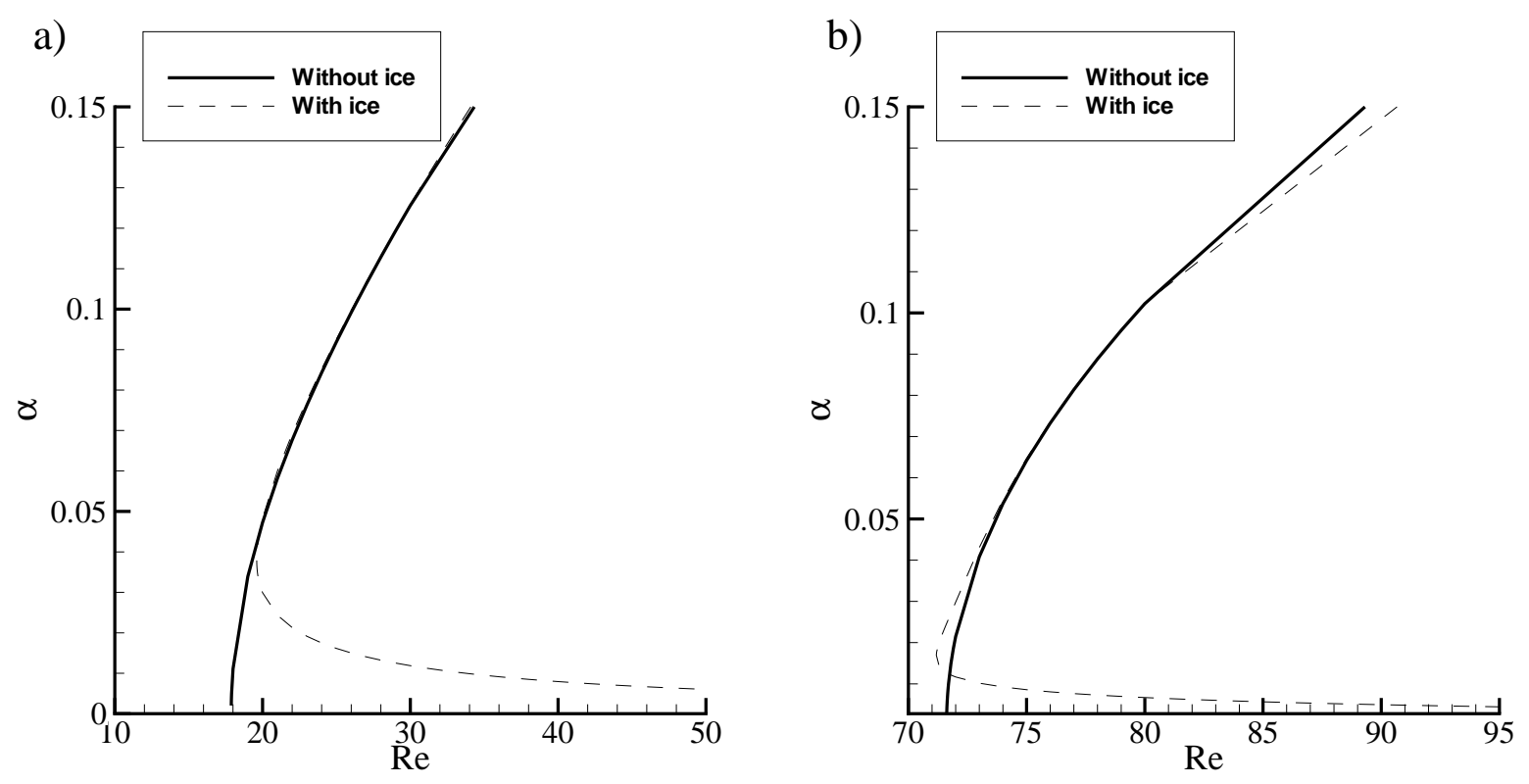

Figure 11: The influence of the ice layer on the surface mode neutral curves $\left(S t=0.0625, H_{0}=10\right)$ : a) $\phi=4^{\circ}$; b) $\phi=1^{\circ}$. 
a)

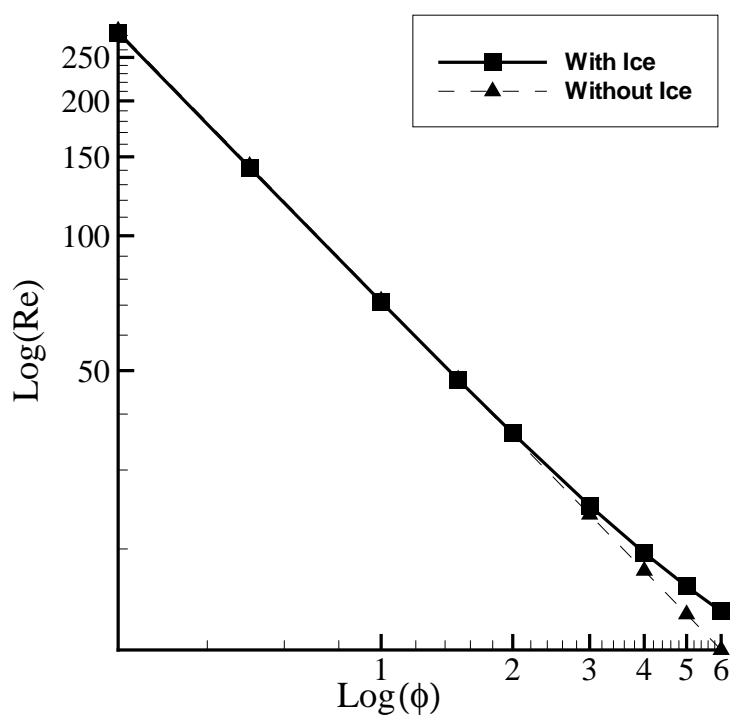

c)

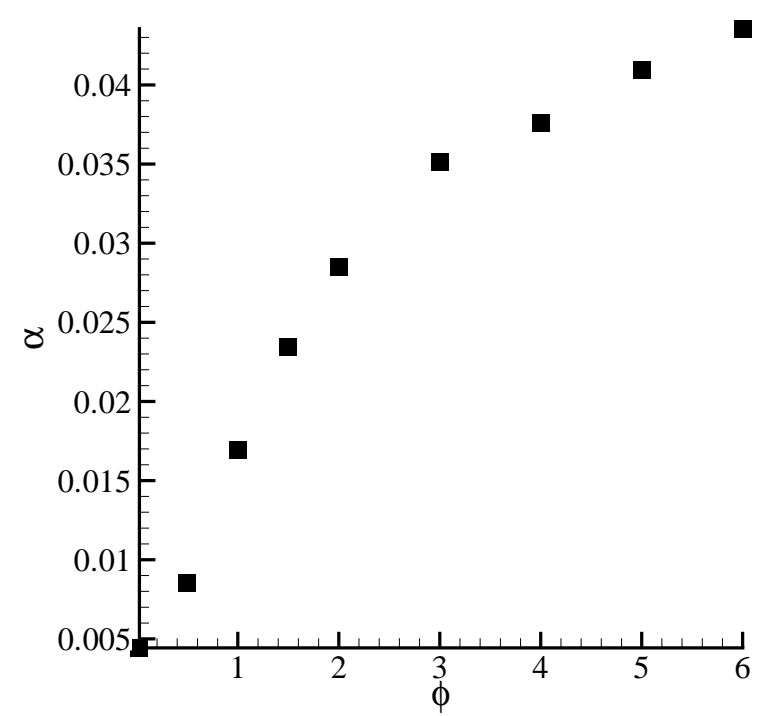

b)

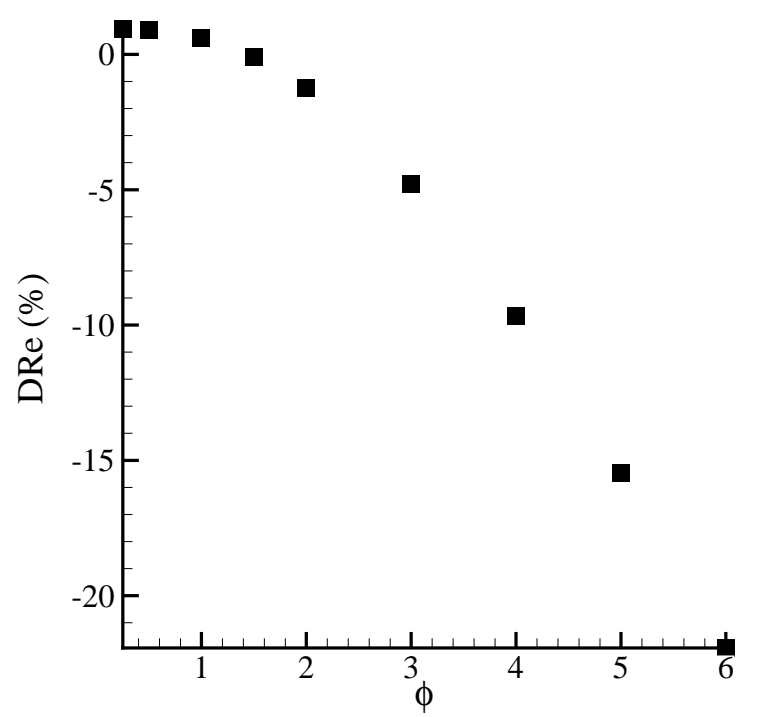

d)

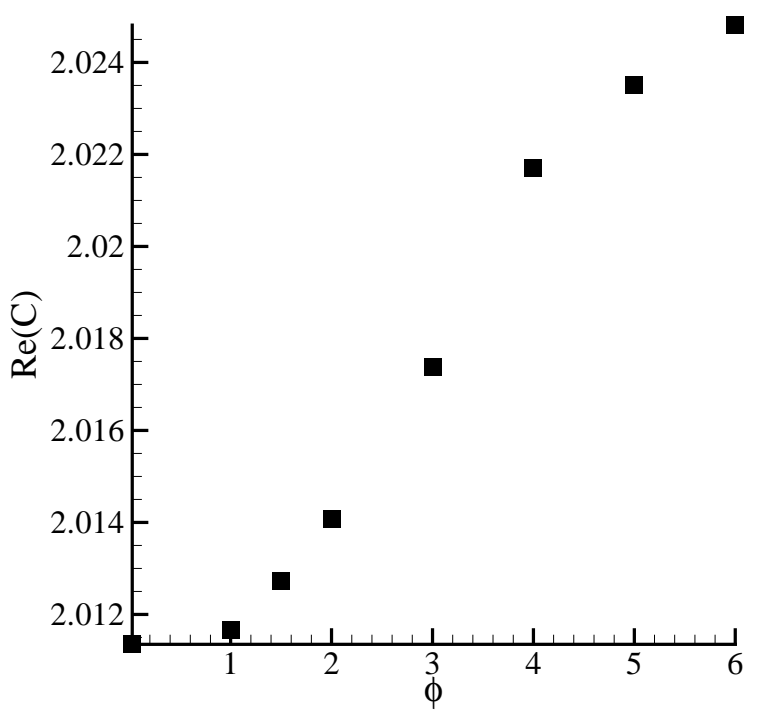

Figure 12: The influence of the angle of incline on the surface mode critical parameters: a) Critical Reynolds; b) Critical Reynolds difference $D R e$; c) Critical wave number; d) Critical phase speed. 